\title{
Hierarchical Organization of Areas in Rat Visual Cortex
}

\author{
Thomas A. Coogan and Andreas Burkhalter \\ Department of Anatomy and Neurobiology, Washington University School of Medicine, St. Louis, Missouri 63110 ri 63110
}

To test the hypothesis that areas within rat visual cortex are organized in a multilevel hierarchy, we have employed Phaseolus vulgaris leucoagglutinin as an anterograde axonal tracer to visualize the laminar patterns of connections between different cortical areas. For identification of cortical areas, we used a combination of markers that included callosal connections, the patterns of inputs and outputs to ipsilateral cortical and subcortical targets, and geographical location. Projections from area 17 to every identified extrastriate target area extend throughout all layers of cortex and include layer 4. Area LM (lateromedial), contained within the cytoarchitectonic subdivision 18 a, projects to area 17 , area $\mathrm{AL}$ (anterolateral), area $\mathrm{RL}$ (rostrolateral), multiple sites within the posterior complex $(\mathrm{PX})$, the anterior complex (AX), the far lateral complex (FLX), the medial complex (MX), perirhinal, entorhinal, retrosplenial, and presubicular cortex. Each of the projections to extrastriate areas resembles those originating from area 17 . Only the projection to area 17 differs, and terminates largely in layers outside of lamina 4. Such projections are designated as feedback (Coogan and Burkhalter, 1990). The projections of a second area, $A L$, of the cytoarchitectonic subdivision $18 \mathrm{a}$ are similar to those of $L M$ : all terminate in layers 1-6, except the inputs to area 17, LM, and a site in FLX, which spare layer 4 . The feedback projection to $L M$ provides further support that $L M$ and $A L$ constitute distinct cortical areas. Projections from additional distinct sites within area 18 a that are located immediately lateral to $L M$ and $A L$ and are designated FLX make feedback projections to area 17 and projections involving all layers to $L M$ and $A L$. Thus, unlike the asymmetrical laminar organization of reciprocal connections between area 17 and $L M, 17$ and $A L$, and $L M$ and $A L$, the connections between $L M$ and at least one site in FLX are symmetrical. Projections that include layer 4 can, therefore, be components of connections between different hierarchical levels as well as components of connections on the same hierarchical level. The MX sites contained within the cytoarchitectonic subdivision $18 \mathrm{~b}$ send feedback projections to striate cortex, LM, AL, and PX within $18 \mathrm{a}$. Thus, the connections between these areas are reciprocal and the laminar organization is asymmetrical. The pro-

\footnotetext{
Received June 15, 1992; revised Mar. 1, 1993; accepted Apr. 7, 1993.

We are grateful to David C. Van Essen and Randy Johnson for valuable comments on the manuscript, and to Vinod Charles and Steve Mitan for excellent assistance with histology and photography. This work was supported by EY05935.

Correspondence should be addressed to Andreas Burkhalter, Department of Anatomy and Neurobiology, Box 8108, Washington University School of Medicine, 660 South Euclid Avenue, St. Louis, MO 63110.

Copyright (C) 1993 Society for Neuroscience $0270-6474 / 93 / 133749-24 \$ 05.00 / 0$
}

jections to FLX include layer 4 , and the projections to frontal, cingulate, and retrosplenial cortex resemble forward projections.

Although the areal organization of extrastriate cortex is not yet fully resolved, using the patterns of intracortical connections we are able to construct a provisional hierarchy of cortical areas. In this scheme, area 17 is at the first level, $L M$ ranks higher and is likely at the second level, $A L$ ranks above $L M$, and $M X$ is at a still higher level. Sites in FLX receive columnar projections from $L M, A L$, and $M X$, and so by our criteria this complex spans the hierarchical levels that these areas occupy. The ordering of cortical areas is consistent with an ordering derived from the pattern of corticotectal projections where inputs from successively higher areas terminate in increasingly deeper layers.

[Key words: intracortical connections, visual cortex, hierarchy, cortical layers, rodent, forward connections, feedback connections]

Visual perception emerges from the encoding of optical features in separate functional channels, and the progressive integration of information in a hierarchy of cortical areas that interact through networks of forward and feedback connections (DeYoe and Van Essen, 1988; Felleman and Van Essen, 1991). Although the general principles of this macrocircuit are relatively well understood, little is known about the reciprocal circuits involved in the transformation of information between different cortical areas. Over the past years, our interest has been to develop the rat visual system as an accessible model for investigating synaptic interactions in reciprocal intracortical circuits. To this end, we began to examine the connections between striate cortex (primary visual cortex, area $17, \mathrm{~V} 1$ ) and surrounding extrastriate areas, which led to the discovery of two distinct projection patterns (Coogan and Burkhalter, 1990). Projections from primary visual cortex give rise to columns of terminations in extrastriate cortex that extend from inner layer 1 to outer layer 6 . In contrast, projections returning from extrastriate cortex to striate cortex lack strong input to layer 4 and preferentially terminate in layers $1,2 / 3,5$, and 6 . Although these patterns are reminiscent of forward and feedback projections in monkey visual cortex (Rockland and Pandya, 1979; Maunsell and Van Essen, 1983), it remained unresolved whether in the rat these connections also occur in reciprocal pairs. Clarification of this issue is critical, because the relative ranking of cortical areas depends on the identification of asymmetrical connectivity patterns between reciprocally connected areas.

It is generally accepted that the rodent visual cortex is subdivided into several distinct areas, but there are different views about the number and organization of these areas. Electro- 
physiological mapping experiments in the rat revealed several complete representations of the visual hemifield bordering striate cortex (Montero et al., 1973a), which correspond to an equivalent number of striate cortical projections into the region (Montero et al., 1973b). Several years later, Cusick and Lund (1981) described a complex pattern of callosal connections within extrastriate cortex, which led to the discovery of a systematic relationship between the callosal input and the projections from the striate cortex (Olavarria and Montero, 1984), adding weight to the suggestion that rat primary visual cortex is bordered by three distinct areas: lateromedial (LM), anterolateral (AL), and posterolateral (PL) (Montero, 1981).

A study of the visuotopic organization of mousc cxtrastriatc cortex (Wagor et al., 1980) revealed a distribution of receptive fields similar to that found in the rat (Espinoza and Thomas, 1983; Thomas and Espinoza, 1987), but these authors drew different conclusions about the organization of areas. In the view of Wagor and colleagues a single area, V2, containing a secondorder transformation of the visual field, wraps around a large portion of striate cortex. This organization is similar to that found in cats and primates, and was taken as evidence that V2, like striate cortex, is homologous in all mammals (Kaas, 1980). A more recent study of visual cortical connections proposed an even larger V2, for the rat (Malach, 1989). Malach reported the tendency of striate cortex to project most prominently to the nearest point of extrastriate cortex, and linked the multiple projections of striate cortex to the patchy connections between $\mathrm{V} l$ and V2 of cats and monkeys (Van Essen et al., 1986; Gilbert and Wiesel, 1989). He therefore proposed that the wholc bclt of extrastriate cortex bordering $\mathrm{V} 1$ is occupied by a single area, V2.

Our own studies of the laminar organization of connections between the cytoarchitectonic subdivisions $17,18 \mathrm{a}$, and $18 \mathrm{~b}$ lead us to doubt that the whole of circumstriate cortex can be regarded as a single visual area (Coogan and Burkhalter, 1990). In particular, we have found asymmetric patterns of connections between lateral (area 18a) and medial (area 18b) extrastriate cortex. Such asymmetric laminar patterns of connections have increasingly been relied upon as an indicator of the hierarchical relationship between distinct areas (Friedman et al., 1986; Andersen et al., 1990; Boussad et al., 1990; Felleman and Van Essen, 1991). To test the idea of a hierarchy of visual areas in rat visual cortex more directly, we examined the projection patterns between several identified visual areas, using a combination of neuronal tracing with Phaseolus vulgaris leucoagglutinin (PHAL; Gerfen and Sawchenko, 1984) and visualization of callosal projections. The results show that asymmetric projections exist within lateral extrastriate cortex in addition to those between lateral and medial extrastriate cortex. This strongly supports the view that rat striate cortex is surrounded by multiple distinct visual areas and that these are arranged in a multilevel hierarchy, analogous to the organization in visual cortex of cats and monkeys (Felleman and Van Essen, 1991).

\section{Materials and Methods}

Adult Long-Evans rats ( $n=25,150-270 \mathrm{gm}$ body weight) were anesthetized for surgery with chloral hydrate $(350 \mathrm{mg} / \mathrm{kg}$, i.p.). Animals were placed in a head holder, and burr holes were made in the skull for injections of tracers into different regions of visual cortex: area 17 (3$3.5 \mathrm{~mm}$ lateral of midline, $0-0.5 \mathrm{~mm}$ anterior to $\lambda$-point; $n=13$ ), $\mathrm{LM}$ $(5.5-6.5 \mathrm{~mm}$ lateral of $\lambda$-point; $n=5)$, AL (6 mm lateral of midline, 1.5-2.5 mm anterior of $\lambda$-point; $n=4$ ), far lateral complex (FLX; 6.5$7 \mathrm{~mm}$ lateral of $\lambda$-point; $n=1)$, and medial complex $(\mathrm{MX} ; n=2)$.
Tracing ipsilateral connections with PHAL in combination with transcallosal labeling with Dil or HRP. PHAL was used for tracing ipsilateral corticocortical projections. Injections were made according to the protocol of Gerfen and Sawchenko (1984): PHAL (Vector, Burlingame, $\mathrm{CA} ; 2.5 \%$ in $0.1 \mathrm{M}$ phosphate buffer) was injected iontophoretically (5 $\mu \mathrm{A}$ positive current, $7 \mathrm{sec}$ on $/ 7 \mathrm{sec}$ off duty cycle, $10-15 \mathrm{~min}$ ) from glass micropipettes (tip diameter, 10-15 $\mu \mathrm{m}$ ). For simultaneous labeling of transcallosal connections in PHAL-injected animals we used axonal tracing with DiI (1,1'-dioctadecyl-3,3,3',3'-tetramethylindocarbocyanine perchlorate; Molccular Probcs; arca 17 injection, $n=1$; LM injection, $n=1$; AL injection, $n=1$ ) or HRP (area 17 injection, $n=$ 8; LM injection, $n=1$; AL injection, $n=1$ ). The transcallosal tracers were injected several days after the PHAL injection, typically $1 \mathrm{~d}$ before animals were killed. DiI ( $10 \%$ in dimethyl formamide) was injected by applying brief pulses of air pressure (Picospritzer, General Valve) to the back of a glass micropipette. Multiple injections $(20-30, \approx 30 \mathrm{nl}$ each) were distributed evenly throughout the occipital and parietal lobes of the hemisphere opposite to the PHAL injection. A similar injection protocol was used in cases in which HRP (Sigma type VI; $10 \%$ in saline) was used to mark the callosal projection.

Five to 14 days after the PHAL injection, animals were perfused with fixatives. Deep anesthesia was induced with pentobarbital $(80 \mathrm{mg} / \mathrm{kg})$ and transcardial perfusion was performed with a rinse of $0.1 \mathrm{M}$ phosphate buffer, followed by $4 \%$ paraformaldehyde in $0.1 \mathrm{M}$ acctatc buffer (pH 6.5), which was followed by $4 \%$ paraformaldehyde in $0.1 \mathrm{~m}$ borate buffer ( $\mathrm{pH} 9.5$ ). Brains were cut on a Vibratome at $50 \mu \mathrm{m}$ in the coronal plane. In cases that were injected with Dil, one series of sections was treated in normal rabbit serum for $1 \mathrm{hr}(2 \%$ in $0.05 \mathrm{M} \mathrm{K-PBS})$ and subsequently incubated in goat anti-PHAL IgG (Vector; 1:2000 preabsorbed against brain tissue, $2 \%$ normal rabbit serum, 0.05 м K-PBS, $0.3 \%$ Triton $\mathrm{X}-100$ ) for $36-48 \mathrm{hr}$ at $4^{\circ} \mathrm{C}$. The primary antibody was visualized with a biotinylated rabbit anti-goat $\operatorname{IgG}(1: 200,2 \%$ normal rabbit serum, 0.05 м K-PBS, $0.3 \%$ Triton X-100), followed by incubation in avidin and biotinylated HRP, which was followed by HRP histochemistry in the presence of diaminobenzidine $(0.002 \%)$ and $0.001 \%$ hydrogen peroxide. Reacted sections were mounted on gelatin-coated slides, air dried, dehydrated in graded alcohols, cleared in xylene, and covcrslipped with Pcrmount. Labcled fibcrs were viewed under darkfield illumination. Selected sections were counterstained with cresyl violet and used to determine laminar (Krieg, 1946), areal, and nuclear borders (Paxinos and Watson, 1986).

In cases whose callosal projection was marked with HRP, PHAL immunocytochemistry was similar, except that prior to PHAL staining the sections were treated with a mixture of methanol (10\%) and hydrogen peroxide $(3 \%)$ to inactivate HRP.

In cases that were injected with DiI, a second series of unstained sections was used for visualizing DiI-labeled callosal projections. For this purpose sections were mounted on glass slides, coverslipped with $0.1 \mathrm{M}$ phosphate buffer, and viewed under rhodamine fluorescence optics. Labeled callosal projections were photographed at low power. To relate PHAL-labeled projections to transcallosal DiI labeling, adjacent immunolabeled and fluorescent sections were photographed at the same magnification. For aligning fluorescence and dark-field images, blood vessels were used as reference marks.

In cases whose callosal projection was marked with HRP, sections that were not immunostained for PHAL were mounted on gelatincoated slides and air dried. HRP histochemistry was performed using tetramethyl benzidine as the chromogen (Mesulam, 1978). The relationship between PHAL-labeled projections and the transcallosal HRP labeling was determined by aligning adjacent sections and reconstruction under a camera lucida.

Combined labeling of ipsilateral connections with PHAL and rhodamine dextran. To determine the topographic organization within different areas of extrastriate cortex, pairs of injections with PHAL and tetramethyl rhodamine dextran-lysine (RD) were made at different points of the map in area 17. For this purpose, PHAL was injected iontophoretically as described above. $\mathrm{RD}\left(10 \%\right.$ in $\mathrm{H}_{2} \mathrm{O}$; Molecular Probes) was injected by pressure $(50 \mathrm{nl})$. After $3-5 \mathrm{~d}$ animals were reanesthetized and the opposite hemisphere was injected with HRP, to label the callosal projection. One day later, animals were perfused with fixatives using the pH-shift protocol outlined above. Subsequently the brain was removed from the skull, and the cortex was dissected and flattened between slides. The cortex was then sectioned $(50 \mu \mathrm{m})$ on a Vibratome in the horizontal plane. Adjacent sections were reacted for PHAI and HRP, or left unstained for photography of RD-labeled projections under 

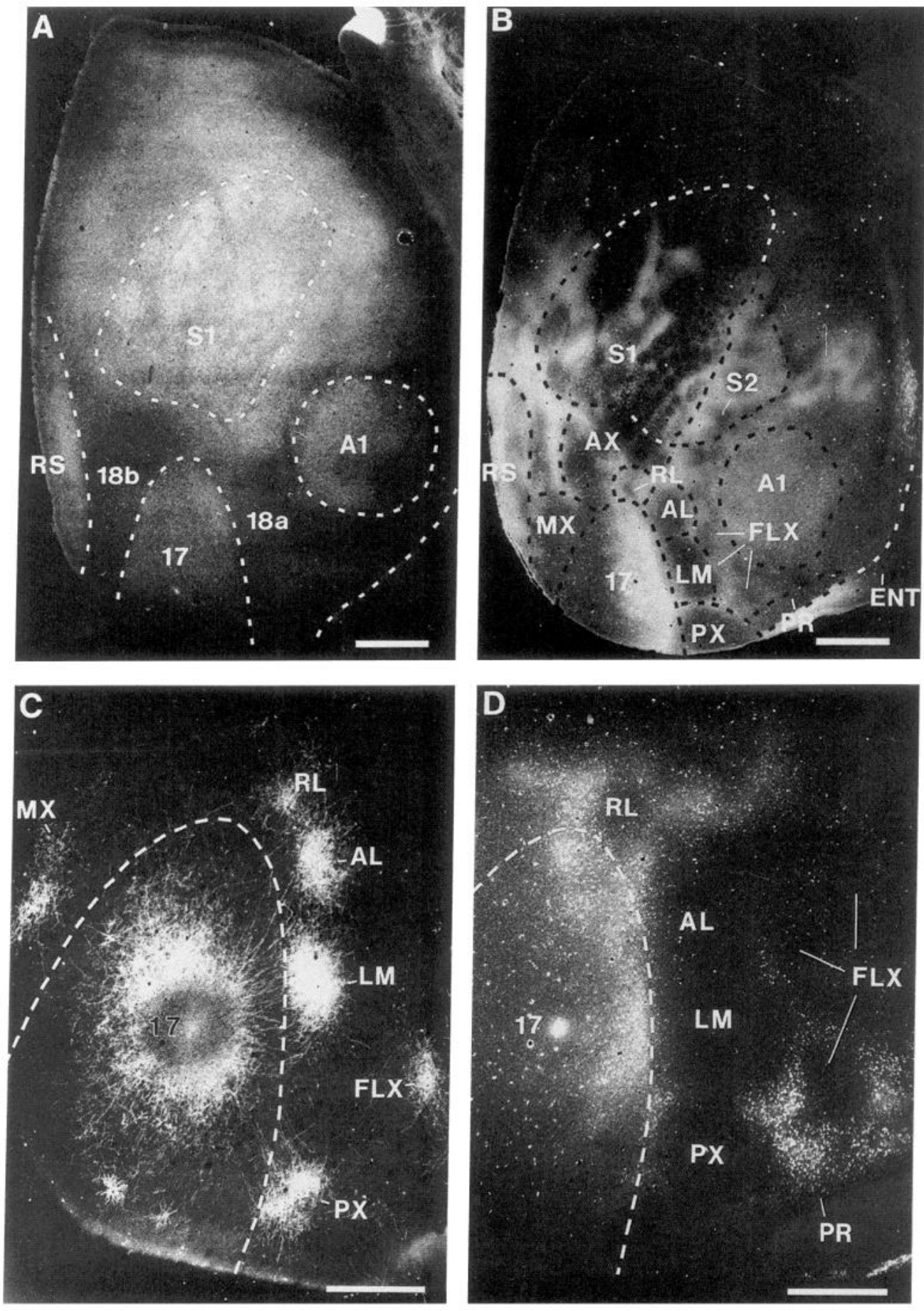

Figure 1. Anatomically defined subdivisions within rat occipital cortex. $A$, Myeloarchitecture of rat cerebral cortex: horizontal section through flattened cerebral cortex, viewed under dark-field illumination. The section is unstained and mounted in phosphate buffer. Light areas represent regions of dense myelination. Weakly myelinated areas appear dark. 17, primary visual cortex. $18 a$ and $18 b$, approximate location of respective cytoarchitectonic areas. $B$, HRP-labeled callosal connections of occipital, parietal, and temporal cerebral cortex: horizontal section viewed under dark-field illumination. Broken lines indicate the approximate borders of identified areas $(17, \mathrm{~A} 1, \mathrm{AL}$, LM, RL, S1; for abbreviations, see Appendix) and several more tentatively assigned cortical subdivisions (AX, ENT, FLX, MX, PR, RS, S2). $C$, Projections of rat area 17: fiber labeling pattern in horizontal section, seen under dark-field illumination, after injection (dark spot in center) of PHAL into area 17. $D$, DiI-labeled callosal connections: horizontal section adjacent to that shown in $C$ viewed under fluorescence illumination. Note that the PHAL-labeled extrastriate projections shown in $C$ terminate in callosal-free zones. Scale bars: $A$ and $B, 2 \mathrm{~mm} ; C$ and $D, 1 \mathrm{~mm}$. the fluorescence microscope. For determining the borders of striate cortex, these unstained sections were also viewed under dark-field optics to reveal the myeloarchitecture. Parallel sections containing each of the different labeling patterns (PHAL, HRP, RD) were reconstructed under a camera lucida and superimposed by aligning blood vessels.

\section{Results}

\section{Nomenclature}

To describe the projections identified in this study, we use a mixture of terms from previous studies as well as introducing some new ones. The terms area 17 and striate cortex are employed to refer to primary visual cortex, which in tangential sections appears as a parabola-shaped region of heavy myelination with its broad base at the posterior pole of the occipital cortex (Fig. 1A; Olavarria et al., 1987). This region closely matches that outlined in physiological mapping studies of primary visual cortex (Montero et al., 1973a; Espinoza and Thomas, 1983). The surrounding extrastriate cortex contained within the cytoarchitectonic subdivisions 18a and 18b (Krieg, 1946; Miller and Vogt, 1984) consists of multiple distinct representations of the contralateral hemifield (Espinoza and Thomas, 1983) that are delineated by a stereotypical pattern of callosal connections (Olavarria and Montero, 1984; Thomas and Espinoza, 1987). We have retained some of the terms introduced 
in these studies in reference to areas within the cytoarchitectonic subdivision $18 \mathrm{a}$ in lateral extrastriate cortex. This includes AL and $\mathrm{LM}$, which refer to the visual representations contained within the anterior and posterior half of the large acallosal oval lateral to striate cortex (Fig. $1 B$ ). It also includes RL (rostrolateral) to refer to a region identified by a ring of callosal input anterior to the acallosal oval (Fig. 1B).

In other parts of cortex adjoining striate cortex, the correlation of physiological and connectional evidence is incomplete and none of the existing terms provide for an unambiguous identification of the particular region of cortex. We have therefore introduced new terms that take account of the complexity that has been demonstrated by connectional studies. One of these regions lies lateral to the acallosal oval and receives multiple projections from striate (Olavarria and Montero, 1984) and extrastriate cortex (present study) that have not yet been clearly associated with callosal borders or visuotopic maps. Because it is unclear whether terms used in the past to describe these projections actually refer to the same sites, we will refer to projections lateral to AL and LM as projections to the FLX (Fig. $1 B$ ). Unequivocal areal definitions are also lacking for territories anterior and medial to striate cortex, and posterior to LM. Anterior extrastriate cortex receives multiple projections from striate cortex, which in different reports have been termed " $A$ " and " $S$ " (Olavarria and Montero, 1984; Malach, 1989). In the medial extrastriate cortex, multiple representations of visual space have been identified (Espinoza and Thomas, 1983), and anatomical studies have revealed multiple clusters of striate cortical projections to this region (Miller and Vogt, 1984; Olavarria and Montero, 1984; Malach, 1989; present study; see Fig. 3). However, the connectional patterns in medial cortex have not yet been related to the physiologically defined topographic maps. Similarly, the cortex posterior to LM receives multiple projections from striate cortex (Olavarria and Montero, 1984; Malach, 1989; present study; see Fig. 3), indicating that this region presumably contains a complex of areas, which have been referred to as posterior (P) or PL areas. Although some topographic information is available for this region (Espinoza and Thomas, 1983), it is incomplete and it remains difficult to correlate projections with specific cortical areas. Thus, for each of these regions we have adopted terms that reflect that they are composed of multiple functional units: AX, for the anterior extrastriate complex contained between area $17, \mathrm{KL}, \mathrm{Sl}$, and the $\mathrm{C}$-shaped callosal band at the medial border (Fig. 1B); MX for the medial extrastriate complex contained between area 17 , the retrosplenial cortex (RS), and the C-shaped callosal band at the lateral border (Fig. $1 B$ ); and posterior complex (PX) for the posterior extrastriate complex contained within the acallosal zone lateral to area 17 and posterior to LM (Fig. $1 B$ ). The definition of these regions is provisional, and each of these names should be con- sidered a temporary, geographic term used only until the functional units of these regions are well delineated.

\section{Projections of striate cortex}

Before discussing the laminar organization of striate cortical projections based on serial reconstructions from coronal scctions, it is helpful to view the projection pattern in horizontal sections. Figure $1 C$ shows the result of an area 17 injection near the center of gaze. Multiple distinct projection clusters surrounded striate cortex. Typically, four projections were aligned along the lateral border of area 17. The most anterior projection terminated in RL, closely associated with the small acallosal ring (Fig. $1 D$ ) at the rostral tip of striate cortex. Behind RL followed the projections to $\mathrm{AL}$ and $\mathrm{LM}$, which both were contained within an acallosal oval (Fig. 1D). Posterior to this lay a third acallosal region that contained projections to PX. Cortex lateral to the acallosal oval and medial to auditory cortex contained a complex of callosally connected and acallosal zones (Fig. $1 B, D$ ) called FLX, a region that always received input from striate cortex. The projection to MX lay in a poorly myelinated region with a complex pattern of callosal input adjoining the medial border of area 17.

Topographic organization of extrastriate projections. To investigate whether some of these striate recipient extrastriate regions contained a topographic organization of projections, we performed double-labeling experiments in which two anterograde tracers were injected at different points in the visuotopic map of area 17. In the experiment illustrated in Figure 2, RD was injected at the medial margin of striate cortex, near the midpoint of its anteroposterior extent (Fig. 2B), while PHAL was injected in posteromedial striate cortex. According to published topographic maps (Montero et al., 1973a; Espinoza and Thomas, 1983), the RD injection site corresponds to a point representing the lower temporal field, whereas the PHAL injection site is mapped in the upper nasal visual field. Within the acallosal oval lateral to area 17 (Fig. 2D), each injection marked two distinct sites: PHAL-labeled fibers were clustered close to the medial border at the anterior and posterior tip (Fig. $2 C, D$ ), whereas RD-labeled clusters occupied more central and lateral positions within the oval (Fig. 2C,D). These labeling patterns agree with physiologically derived topographic maps (Montero et al., 1973a; Espinoza and Thomas, 1983; Thomas and Espinoza, 1987) and support the notion that the acallosal oval contains at least two distinct areas, AL and LM.

Figure 3 provides a more complete view of the topographic organization within extrastriate cortex by showing the results of three additional double-labeling experiments (Figs. $2 A-D$ and $3 A$ illustrate same case). The most important feature that is evident from Figure 3 is that each injected point of striate cortex made projections to multiple sites found in all parts of

\footnotetext{
Figure 2. Topographic organization of extrastriate areas LM and AL revealed by anterograde tracing, with PHAL and RD, of projections from different points in the visuotopic map of area 17. $A-D$ illustrate sections from the same case. $A$, Dark-field photomicrograph of horizontal section through the right hemisphere. Opaque regions represent areas of dense myelination. Transition in myeloarchitecture (arrows) was used to delineate borders of area 17 shown in $B$ and $C . B$, Low-power fluorescence photomicrograph of horizontal section through occipital cortex, showing location of PHAL (white asterisk) and RD (black asterisk) injection sites in area 17. Dark patches in extrastriate cortex lateral to area 17 border represent PHAL-labeled projections to LM and AL. C, Relative positions of PHAL-labeled (white asterisk) and RD-labeled (black asterisk) terminal fields

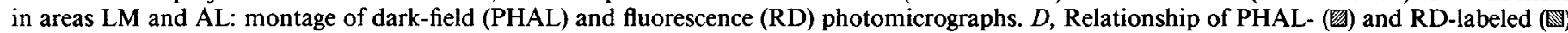
projections in LM and AL to callosal recipient zones marked by HRP. HRP-labeled regions contain cells and terminals and appear white. Broken lines demarcate acallosal oval. Notice that the oval contains two areas, $\mathrm{LM}$ and $\mathrm{AL}$, that both contain a representation of the upper nasal and lower temporal visual field. For abbreviations, see Appendix. In $A-D$, posterior is toward the bottom, lateral toward the right. Scale bars: $A$, 2 mm, $B, 1 \mathrm{~mm}, C$ and $D, 0.5 \mathrm{~mm}$.
} 

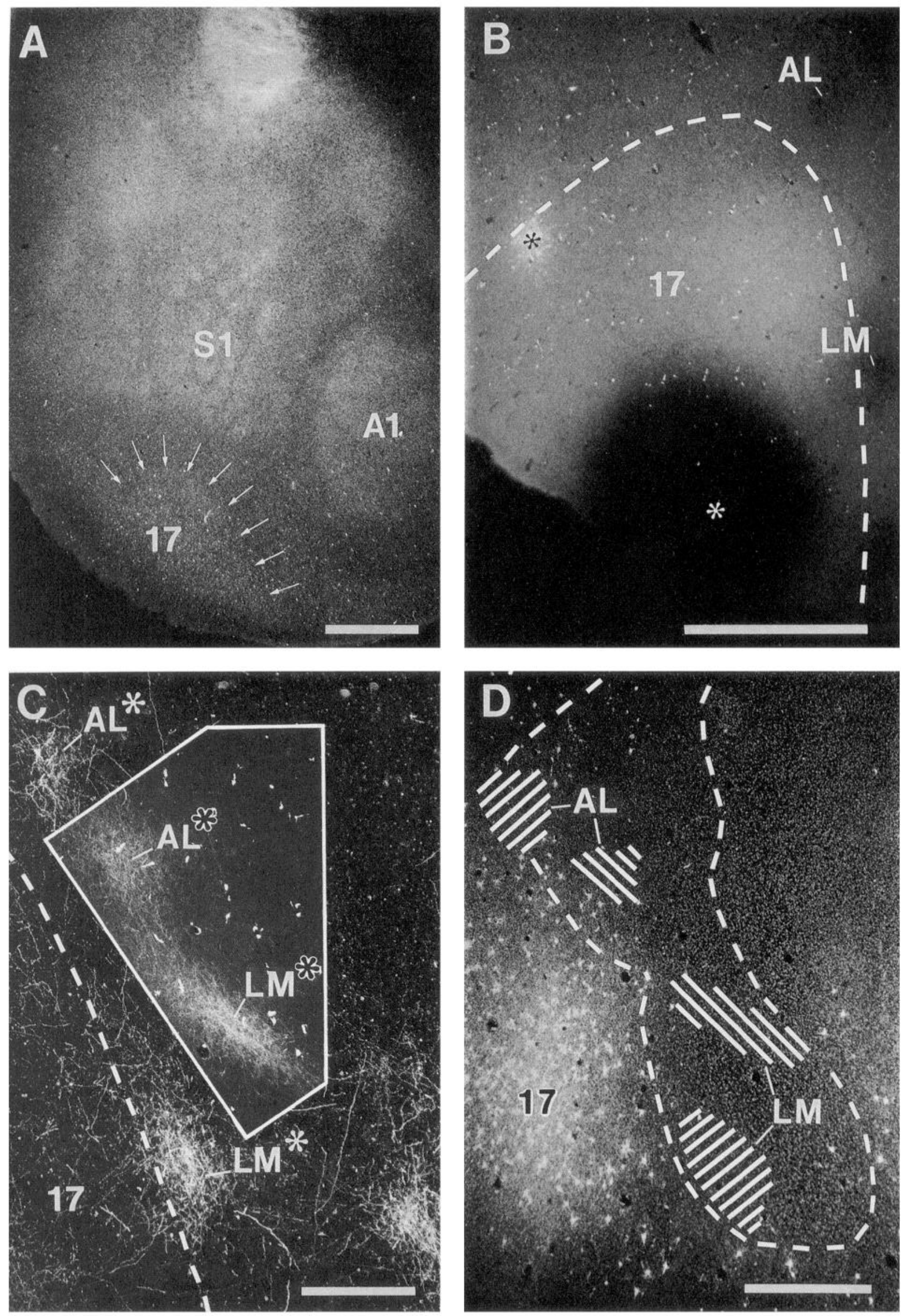
A

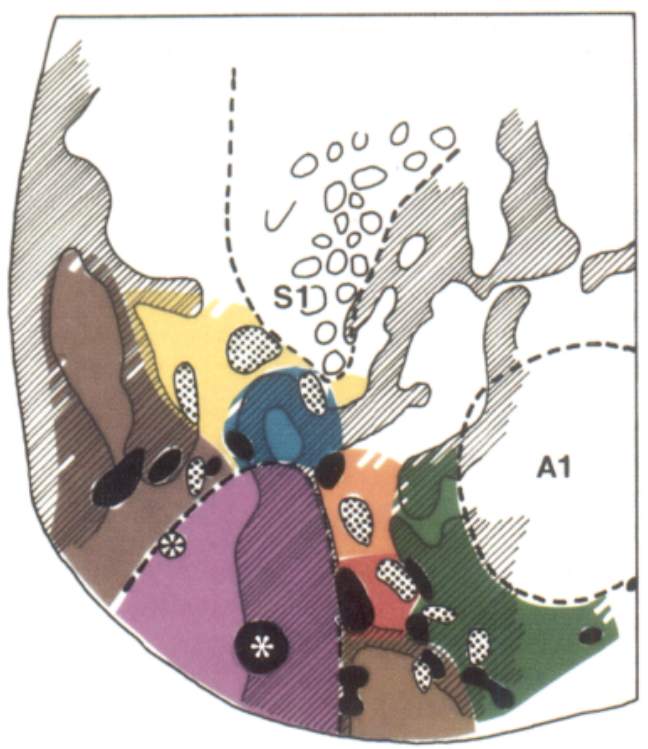

C

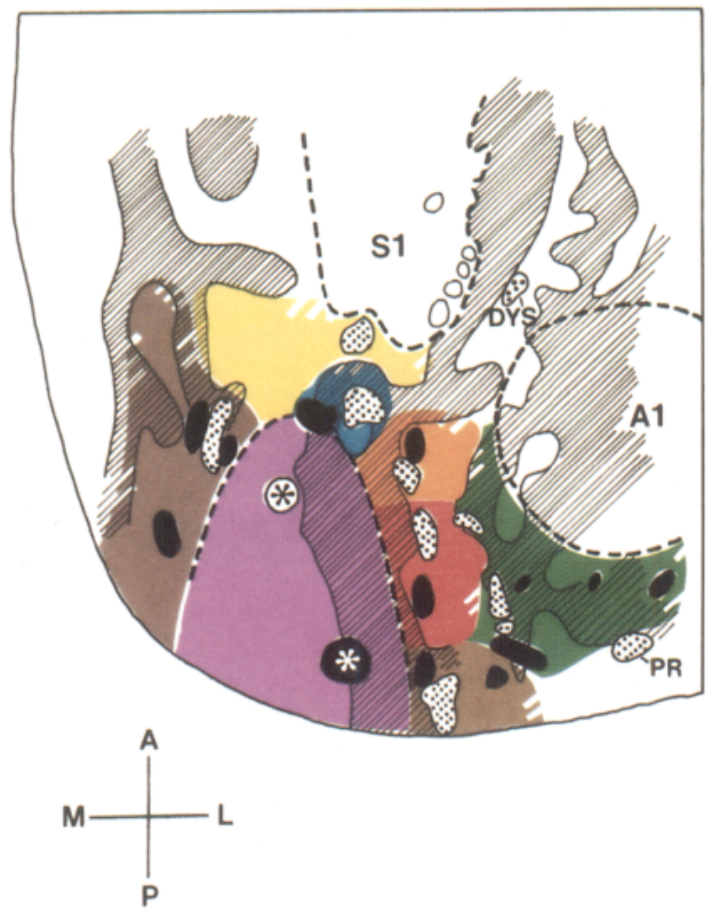

B

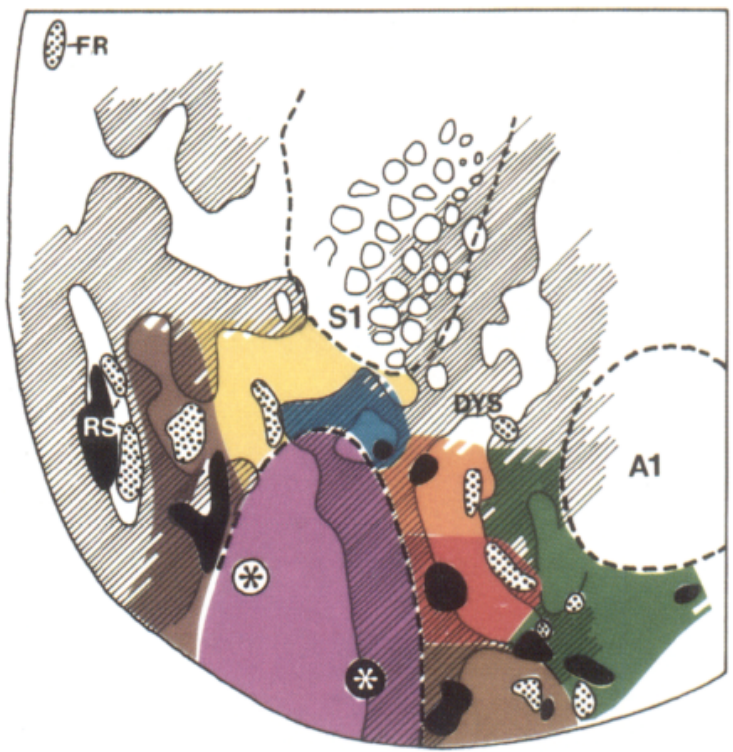

D

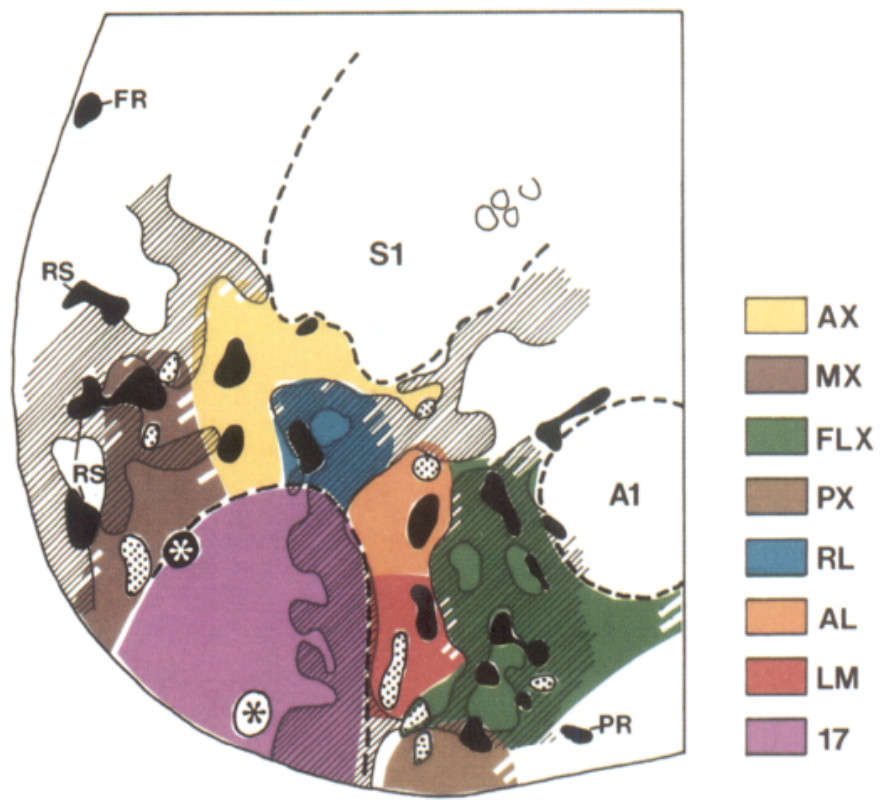

Figure 3. Topographic organization of rat extrastriate cortex, revealed by anterograde tracing with PHAL and RD, of projections from different points in the visuotopic map of area 17. A-D represent reconstructions of horizontal sections of four different cases. White asterisks, PHAL injection sites; black areas, PHAL projection sites; black asterisks, RD injection sites; stippled areas, RD projection sites; hatched areas, HRP-labeled callosal projections. Broken lines represent myeloarchitectonic borders of primary sensory areas. Colored regions represent the approximate (indicated by shredded outlines) territory of distinct areas or subdivisions of extrastriate cortex. For abbreviations, see Appendix. Scale bar: 2 mm.

extrastriate cortex adjoining area 17, except to AX. Specifically, injections placed in medial striate cortex labeled projections in lateral extrastriate cortex, and injections in lateral striate cortex labeled projections in medial extrastriate cortex. Likewise, injections placed in anterior or posterior area 17 labeled projections in posterior and anterior extrastriate cortex, respectively. Thus, the projections were not confined to small regions of extrastriate cortex that are closest to the striate cortical injection site (Malach, 1989). In addition, the arrangement of labeled clusters strongly supports reports of multiple representations of visual space in extrastriate cortex bordering area 17 (Montero et al., 1973a; Espinoza and Thomas, 1983; Thomas and Espinoza, 1987). This is demonstrated most clearly for the acallosal oval lateral to area 17. As discussed for Figure 2, each injection in each case led to two labeled clusters within this region. The results of similar experiments are shown in Figure $3, B$ and $D$ : the two injections were again displaced from each other mediolaterally at comparable anteroposterior positions, putting the 
two injections into different quadrants of the visuotopic map. $A$ reversal was seen in the positions of $A L$ and $L M$ projections labeled by each injection: lateral area 17 injections labeled medial sites, medial injections labeled lateral sites, anterior injections labeled neighboring points in the center of the oval, and posterior injections labeled distant sites at the antcrior and posterior tip of the oval. Figure $3 C$ shows a case in which the two striate cortex injections were displaced anteroposteriorly, along the lower-upper field axis. In this case the AL and LM projections fell roughly along the anteroposterior axis, with the projections from the lower field injection (RD) lying between the projections labeled by the upper field injection (PHAL). These results show that the acallosal oval contains two orderly representations of large parts of the visual field. Thus our findings confirm predictions derived from physiological maps (Espinoza and Thomas, 1983; Thomas and Espinoza, 1987) and support the notion that AL and LM are distinct visual areas.

Outside of $\mathrm{AL}$ and $\mathrm{LM}$, the double-labeling experiments revealed some topographic order in additional regions of extrastriate cortex. In the posterior extrastriate cortex, Thomas and Espinoza (1987) have shown that nasal fields map along the border with striate cortex. As predicted from these maps, Figure $3, A$ and $B$, shows that lateral area 17 injections labeled projections closer to the border of striate cortex than the more medial injections. In addition, upper field injections labeled anterior sites within PX whereas lower field injections marked posterior portions of PX (Fig. 3C). Each case illustrated in Figure 3 also shows nasal representations at the lateral border of the posterior acallosal zone (PX). This suggests the possibility of an additional visual area within PX, but in the face of the variability in our results we remain uncertain about its precise topographic organization.

A clear visuotopic plan was not readily apparent in the region we term FLX. We found a trend that injections of temporal fields produced more labeled fields than injections of nasal representations, but the number of projections was variable. Moreover, we did not obtain clear evidence for a reversal in the arrangement of clustcred projections within the acallosal oval that was predicted from the results of Thomas and Espinoza (1987). They suggested that an area LI is contained within the acallosal oval that was revealed by a temporal to nasal shift at the lateral edge of the oval. Although all cases (Fig. $3 A-D$ ) showed a nasal projection to the lateral edge of the acallosal oval, temporal projections, if present, tended to lie lateral to the nasal projections and were fully contained within the lateral callosal recipient zone (Fig. $3 A-C$ ).

The medial extrastriate cortex showed substantial variability in the number and position of projections labeled by the different injections, and we found little correspondence with reports of the visuotopic organization of this region (Wagor et al., 1980; Espinoza and Thomas, 1983). Specifically, we did not observe a reversal from temporal to nasal to temporal along the anteroposterior axis. Neither did we find projections corresponding to a mediolateral arrangement of upper and lower visual fields. In fact, in all four cases illustrated in Figure 3, nasal upper field injections consistently labeled the most posterior projection within MX.

Consistent projections were seen to a region anterior to striate cortex that seemed closely associated with the anterior callosal ring. Considerable uncertainty, however, exists about the borders and topographic organization of this putative extrastriate area, RL (Thomas and Espinoza, 1987). Two cases showed in- puts from upper and lower fields (Fig. $3 A, C$ ), whereas two others revealed only inputs from upper (Fig. $3 B$ ) or lower (Fig. 3D) fields.

Projections to the extrastriate cortex, $\mathrm{AX}$, bordering anterior area 17 that were not associated with the anterior callosal ring, consistently mapped lower visual ficlds. Thus, it is possible that sites within AX contain incomplete maps of the visual field.

Laminar organization of extrastriate projections. All area 17 injections showed projection patterns similar to that illustrated in Figure 4. In this case the injection was placed in lateral striate cortex, near the midpoint of the anteroposterior extent of area 17. The position of the corticotectal projection in the center of the anterior half of the superior colliculus indicates that the injection was into the representation of the center of gaze (Siminoff et al., 1966) and thus corresponds to the injection shown in Figure $1 C$. Six principal cortical projections were labeled by this injection: one medial to striate cortex and five in anterior and lateral extrastriate cortex. Although Figure 4 represents a serial reconstruction, it is not readily apparent that the lateral projections are to several distinct sites, as shown in Figures $1 C$ and 3 . This is because we have limited the number of sections to a selection that best illustrates the laminar organization of the projections. Sections between projection sites that do not contain labeled fibers are often not shown. The topographic location and the relative position of these projections closely correspond to those described previously (Montero et al., 1973a; Olavarria and Montero, 1984; Malach, 1989). Thus, although callosal markers are unavailable in this particular case, we are confident that the medial projection is part of the MX (Fig. $4 L 2$ ), the most anterior projection in lateral cortex corresponds to RL (Fig. $4 L 1$ ), posterior to that is AL (Fig. 4L2), followed by LM (Fig. $4 L 4$ ) and PX (Fig. $4 L 8$ ). The projection to the FLX (Fig. $4 L 8$ ) is seen lateral to $\mathrm{LM}$ and AL. In addition to these projections, terminations are seen in perirhinal cortex (PR; Fig. $4 L 8$ ) and in the presubiculum (PS; Fig. $4 L 7$ ). Although striate cortical projections to the PS have long been suspected, previous reports (Nauta and Bucher, 1954; Montero, 1981) did not provide unequivocal evidence for terminations in layer 1 (Fig. 4H7), lamina externa, and lamina dissicans of Krieg (1946).

Each of the projections to areas surrounding striate cortex had the same laminar form: at the core of the projection, fibers extended in a solid column from upper layer 6 to deep parts of layer 1. The degree of involvement of layer 1 was variable: it was greatest in LM and AL, and least in MX and RL. Typically, the projections to MX, AL, LM, PX, and FLX were elongated along the anteroposterior axis (Figs. $1 C, 3$ ) and resembled an hourglass that was slightly narrower in layer 4 than in layers 1 , $2 / 3$, and 5 .

Projections within striate cortex extended nearly $3 \mathrm{~mm}$ in the anteroposterior axis and approximately $1 \mathrm{~mm}$ mediolaterally (Figs. 1C, 3, 4L3-L6; Burkhalter and Charles, 1990). The lamination of these intrinsic projections closely resembled the lamination of feedback projections to striate cortex from $\mathrm{AL}$ and LM in lateral extrastriate cortex (Coogan and Burkhalter, 1990). Labeled fibers were dense in layer 5 and layers $2 / 3$ and 1 , but the core of layer 4 was devoid of terminal ramifications (Fig. $4 H 6 b)$.

\section{Projections of $L M$}

Injections $\approx 6 \mathrm{~mm}$ lateral to the $\lambda$-point fell into the posterior part of the callosal-free oval in the lateral extrastriate cortex that contains LM. Without exception, injections resulted in la- 

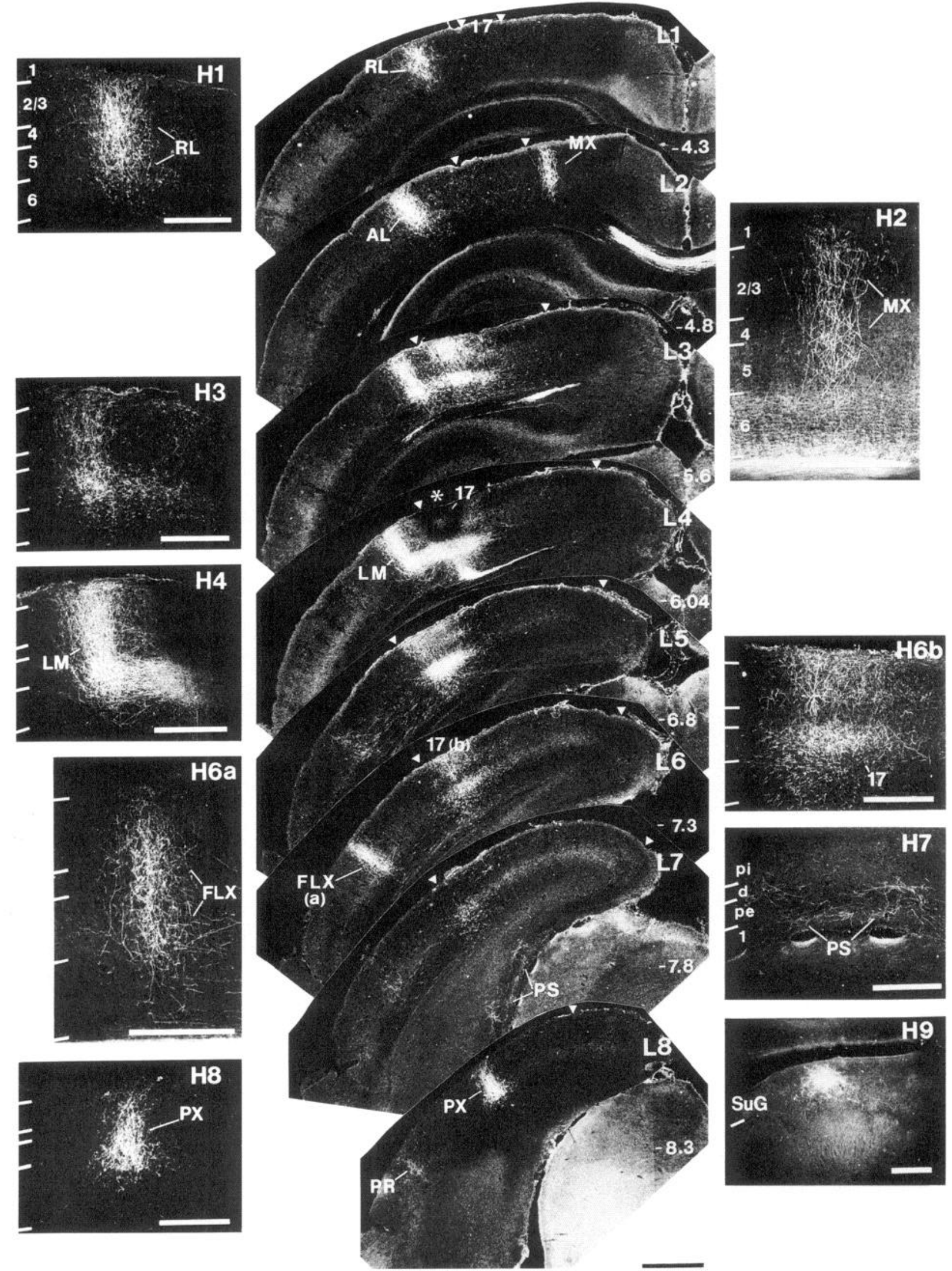

Figure 4. Laminar patterns of PHAL-labeled intracortical projections originating from area 17: reconstruction from serial coronal sections viewed under dark-field illumination. $L 1-L 8$ are low-power photomicrographs (scale bar, $1 \mathrm{~mm}$ ). Numbers on the right indicate approximate distance from bregma in millimeters, as assessed by the closest matching section to that shown by Paxinos and Watson (1986). Asterisk, PHAL injection site; arrowheads, borders of area 17. Hl- H9 are High-power photomicrographs of selected projections (scale bars, $0.5 \mathrm{~mm}$ ). Tick marks indicate laminar borders. For abbreviations, see Appendix. Anterior toward the top, medial to the right. 

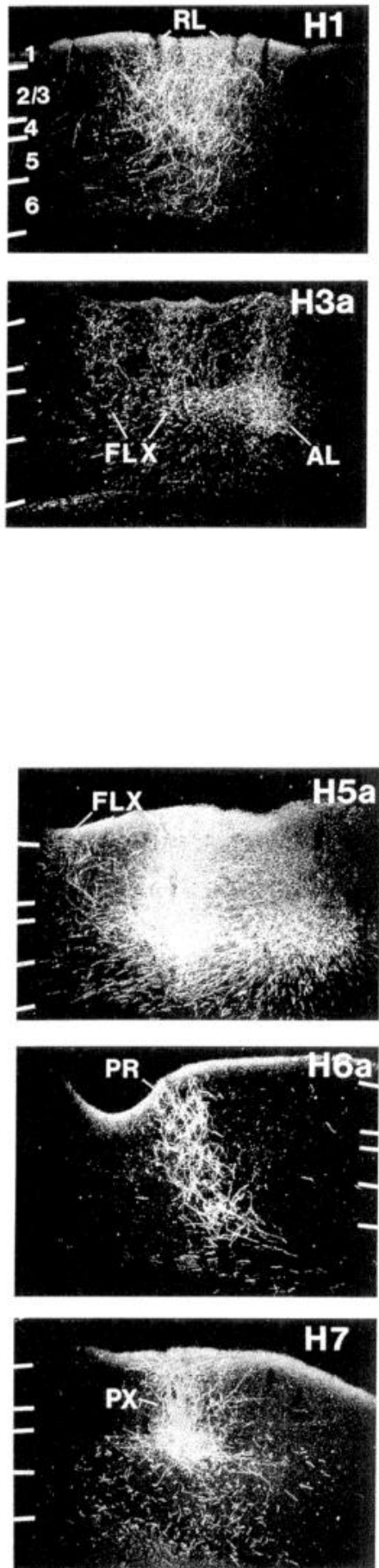
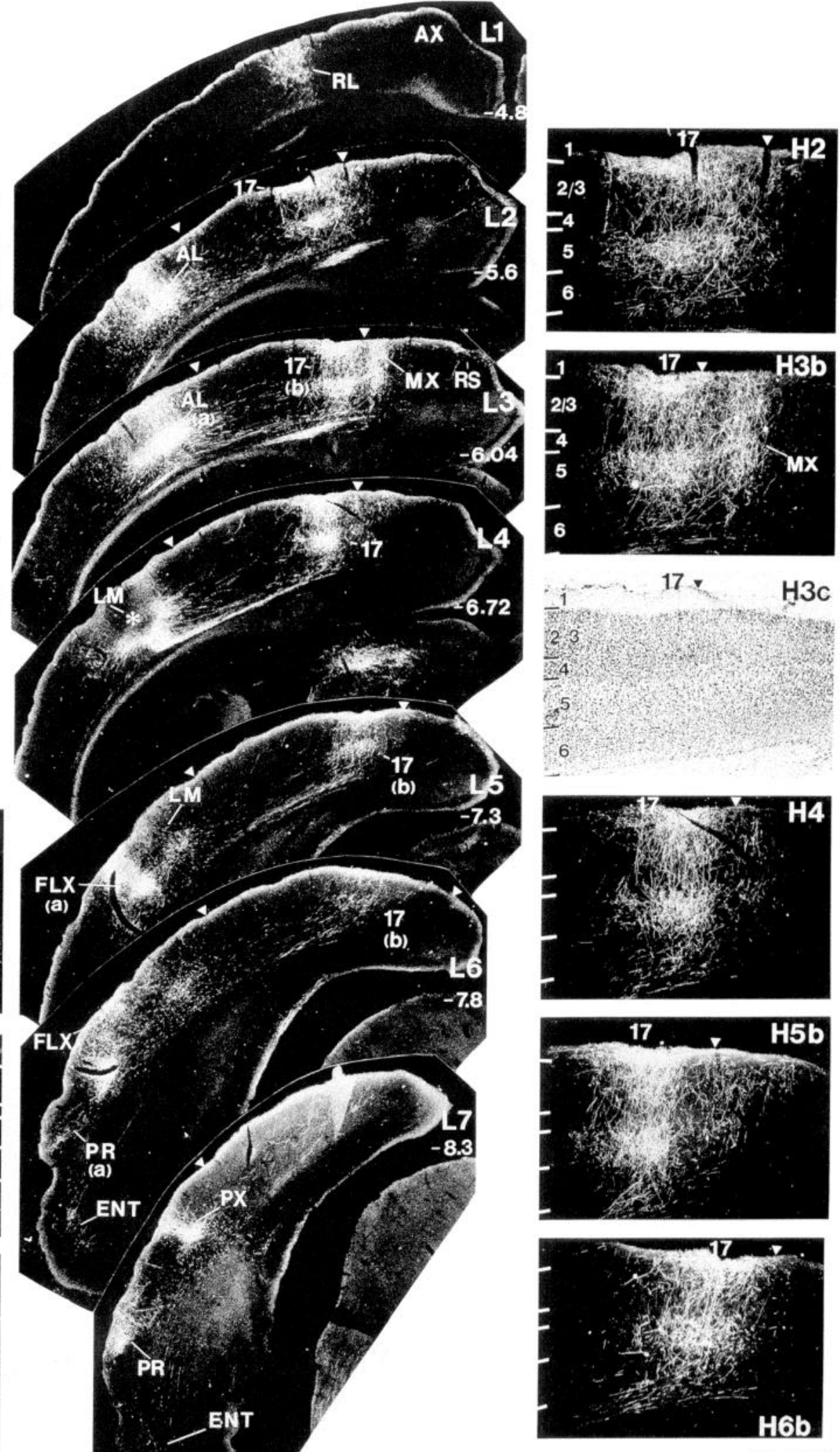

Hob

Figure 5. Laminar patterns of PHAL-labeled intracortical projections originating from area LM: reconstruction from serial coronal sections viewed under dark-field illumination. Injection site is indicated by asterisk. Format, orientation, and scales are the same as in Figure 2. For abbreviations, see Appendix. 

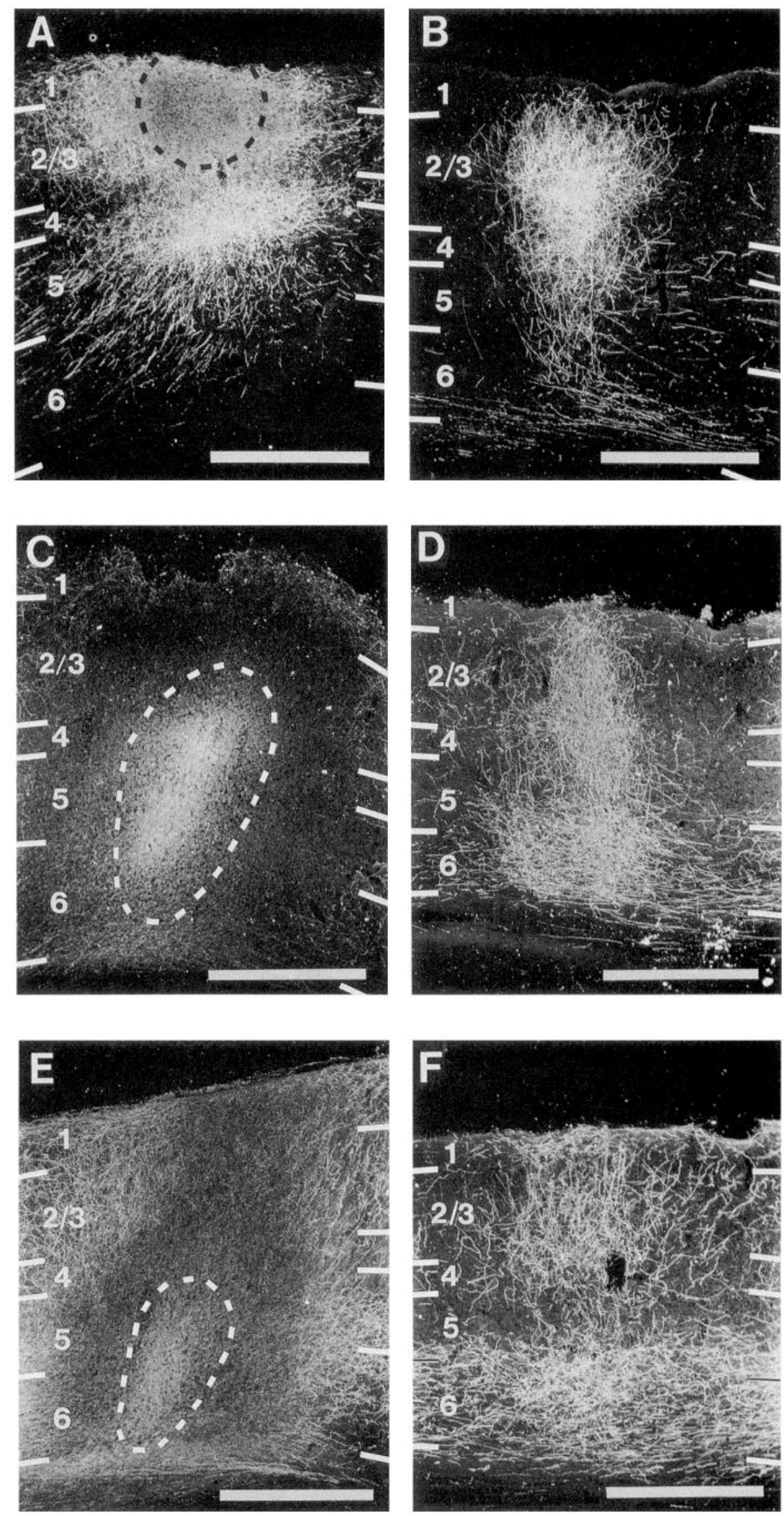

Figure 6. Dependence of projection pattern in LM on laminar position of PHAL injection in area 17: coronal sections. $A$, Dark-field photomicrograph of superficial injection into area 17. Broken line indicates center of injection marked with densely labeled cell bodies. $B, \mathrm{LM}$ projection resulting from injection shown in $A$. $C$, Area 17 injection involving layers $2 / 3-6 . D$, LM projection from injection shown in C.E. Area 17 injection involving mainly layers 5 and $6 . F, \mathrm{LM}$ projection from injection shown in $E$. Scale bars, $0.5 \mathrm{~mm}$. 
beling of dense projections to striate cortex, to RL (Fig. 5L1), AL (Fig. 5L2,L3), and PX (Fig. 5L7) in lateral extrastriate cortex, and to MX (Fig. 5L3) and AX (Fig. 5LI) in medial and anterior extrastriate cortex, respectively. In addition, we also found projections to FLX (Fig. 5L2-L6), PR (Fig. 5L6,L7), entorhinal cortex (Fig. 5L7), RS (Fig. 5L2), PS (Fig. 5L6), frontal cortex (not shown), cingulate cortex (not shown), and the second somatosensory area $\mathbf{S} 2$ (not shown). The projections to FLX extended over an elongated region that appears to contain several distinct projection sites. The most prominent was seen as a column of dense terminations (Fig. 5L5) and presumably corresponds to area LL, identified by Olavarria and Montero $(1981,1984)$. Several sparser projections terminated lateral (Fig. $5 \mathrm{H} 5 \mathrm{a}$ ) and anterior (Fig. $5 \mathrm{H} 3 \mathrm{a}$ ) to the principal projection column. A similar picture was seen in MX, where the main projection column (Fig. $5 \mathrm{L3}, \mathrm{H3} \mathrm{b}$ ) was surrounded by sparser and more diffuse projections (Fig. $5 H 5 b$ ), indicating that MX contains multiple projection sites.

Although in the case shown in Figure 5 we have relied on geographical relationships for areal identification, it is important to stress that the relative positions of the termination sites are in good correspondence with the reported visuotopic arrangement of visual areas, and support our areal assignments. In Figure 4 , the anteromedial position of the projection to striate cortex (Fig. 5L2-L5) and the posterolateral termination of the corticotectal input (Fig. 5L4) indicated that the injection was into the lower, temporal visual field representation (Siminoff et al., 1966; Montero, 1981). This corresponds to a point at the anterior tip of LM (Espinoza and Thomas, 1983). The topographically matching point in AL lies in the posterior part of the area, near the border to LM (Espinoza and Thomas, 1983). This predicts that the AL projection should terminate near the injection site, with little space between the two. Figure $5 L 2-L 4$ shows that this was indeed the case. In contrast, the topographically corresponding point in PX lies at the posterior pole of the occipital cortex (Thomas and Espinoza, 1987) and thus, far away from the LM/PX border. This is precisely what we observed in Figure $5 L 4-L 7$, which showed a $1.5-\mathrm{mm}$ wide gap between the injection site and the PX projection. Finally, the projections to $\mathrm{RL}$ and $\mathrm{AX}$ fit the expectation because both areas are known to possess a representation of the lower visual field (Montero, 1981).

The laminar forms of the visual projections labeled after LM injections were of two types. Projections to extrastriate areas RL (Fig. 5H1), AL (Fig. 5H3a), PX (Fig. 5H7), MX (Fig. 5H3b), and FLX (Fig. $5 H 5 a$ ) had the form of a solid column of terminations extending from the layer $5 / 6$ border to layer 1 , similar to the laminar pattern of projections originating from striate cortex (Fig. $4 H 1, H 2, H 4$ ). In contrast, the projection to striate cortex was concentrated in deep and superficial layers and extremely few terminal ramifications were seen in layer 4 (Coogan and Burkhalter, 1990). The labeled fibers around the injection site in LM were focused deep and superficial to layer 4 (Fig. $5 L 5$ ), and their distribution resembled that of local projections within striate cortex (Fig. $4 \mathrm{H6} 6$ ). Other, sparser projections had less discrete laminar forms. Some of the FLX projections, away from the main projection column, were concentrated in layer $2 / 3$, with fewer terminations in other layers (Fig. $5 H 3 a, L 6$ ). A similar appearance was found in some of the projections within MX (Fig. . $H$ H 5 b).

Projections to nonvisual cortical areas had a variety of laminar forms. The PR projection was like the columnar projections to extrastriate areas and extended evenly through all layers (Fig. $5 H 6 a)$. The entorhinal, frontal, and cingulate projections terminated in layer 1 , in the poorly segregated layers 3 and 4 , and in layer 6 (Krieg, 1946). The projection to RS was largely restricted to layer 1 (Fig. 5L2), as was the PS projection (Fig. 5L6) and the projection to the second somatosensory cortex, though each of these projections also terminated sparsely in deep layers.

\section{Dependence of projection patterns on depth of injection}

The dark brown core of all of our area 17 and LM injections included layers $2 / 3-5$. Lighter staining always reached throughout the thickness of cortex, but since in each case we observed labeling in the thalamus (see Fig. 10), we assume that neurons in layer 6 also transported the tracer. Nevertheless, we were compelled to determine whether the asymmetric labeling patterns observed between area 17 and LM was attributable to differences in the layers included in the injection. Thus, in a series of experiments injections in area 17 and LM were focused in either superficial, middle, or deep layers.

The laminar distribution of labeled terminals in LM was clearly dependent on the depth of injection in area 17. After injections that included layers 1 and $2 / 3$, but not layer 4 , the projection to LM terminated mainly in layers $2 / 3$ and 4 , and relatively few terminations were found in layers 1,5 , and 6 (Fig. 6A, $B$ ). In contrast, all layers were densely labeled when the injections were centered in layers 4 and 5 of area 17 (Fig. 6C,D). Injections centered in layers 5 and 6 of area 17 most strongly labeled layers $1,2 / 3,4$, and 6 while making a very weak projection to layer 5 (Fig. $6 E, F$ ). Importantly, together the contributions from all layers add up to the columnar projections that are illustrated in Figure 3 and that always included layer 4 .

By contrast, the lamination pattern of the projection from LM to area 17 was much less dependent on the depth of the injection. Irrespective of whether injections were centered in superficial (Fig. $7 A, B$ ) or deep layers (Fig. $7 E, F$ ), labeled terminals were always found in layers $1,2 / 3,5$, and 6 , and thus labeling reflected the basic pattern after injections of all layers (Fig. $7 C, D$ ). There was, however, a trend that deeper injections labeled deep layers more densely than superficial layers. Labeling of deep layers from superficial injections was always weak.

\section{Projections of $A L$}

Injections aimed $2.5 \mathrm{~mm}$ anterior to the $\lambda$-point and $6 \mathrm{~mm}$ lateral to the midline were recovered in the anterior portion of the callosal-free oval in lateral extrastriate cortex. In the case shown in Figure 8, the injection was centered in anterior AL where the upper visual field is represented. It resulted in multiple projections to topographically corresponding points in posterior striate cortex (Fig. $8 \mathrm{H} 9$ ), and to LM in posterolateral extrastriate cortex (Fig. $8 L 7-L 9$ ). In addition, $\mathrm{AL}$ was also connected to PX (Fig. 8L9), FLX (Fig. 8L4-L7), MX (Fig. 8L3,L4), RL (Fig. 8L3), AX (Fig. 8Ll), anterior and posterior sites within PR (Fig. 8L9), the second somatosensory cortex (S2; Fig. 8L2), RS (not shown), and the PS (not shown). All of these regions also received input from LM. However, unlike LM, AL projected to the barrel cortex (S1; Fig. 8L2,H2a). The results of upper field $\mathrm{AL}$ and lower field $\mathrm{LM}$ injections indicate that all of the labeled areas contain representations of large portions of the visual field. In addition, they show that $\mathrm{AL}$ and $\mathrm{LM}$ make different intracortical connections, which supports the view that AL and LM are distinct visual areas. Further support for this notion derives from the laminar organization of outputs from AL. In contrast 

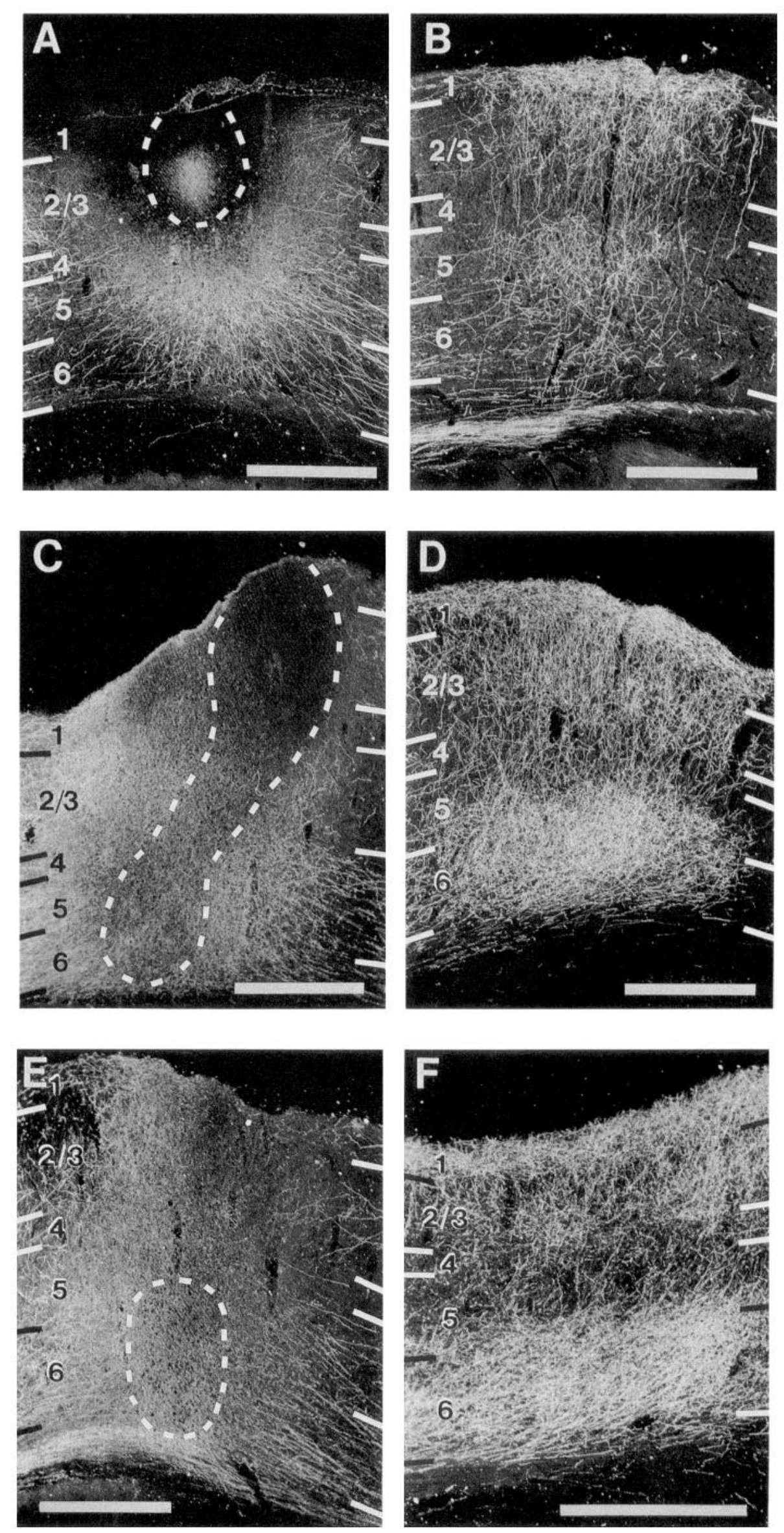

Figure 7. Dependence of projection pattern in area 17 on laminar position of PHAL injection in LM: coronal sections. $A$, Dark-field photomicrograph of superficial injection into LM. Broken line indicates center of injection marked with densely labeled cell bodies. $B$, Area 17 projection resulting from injection shown in $A$. $C, \mathrm{LM}$ injection involving layers 1-6. $D$, LM projection from injection shown in $C$. $E$, LM injection involving layers 5 and $6 . F$, LM projection from injection shown in $E$. Scale bars, $0.5 \mathrm{~mm}$. 

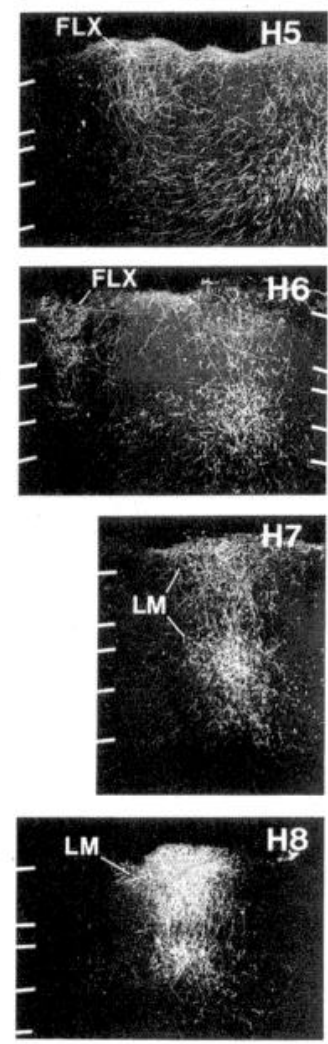
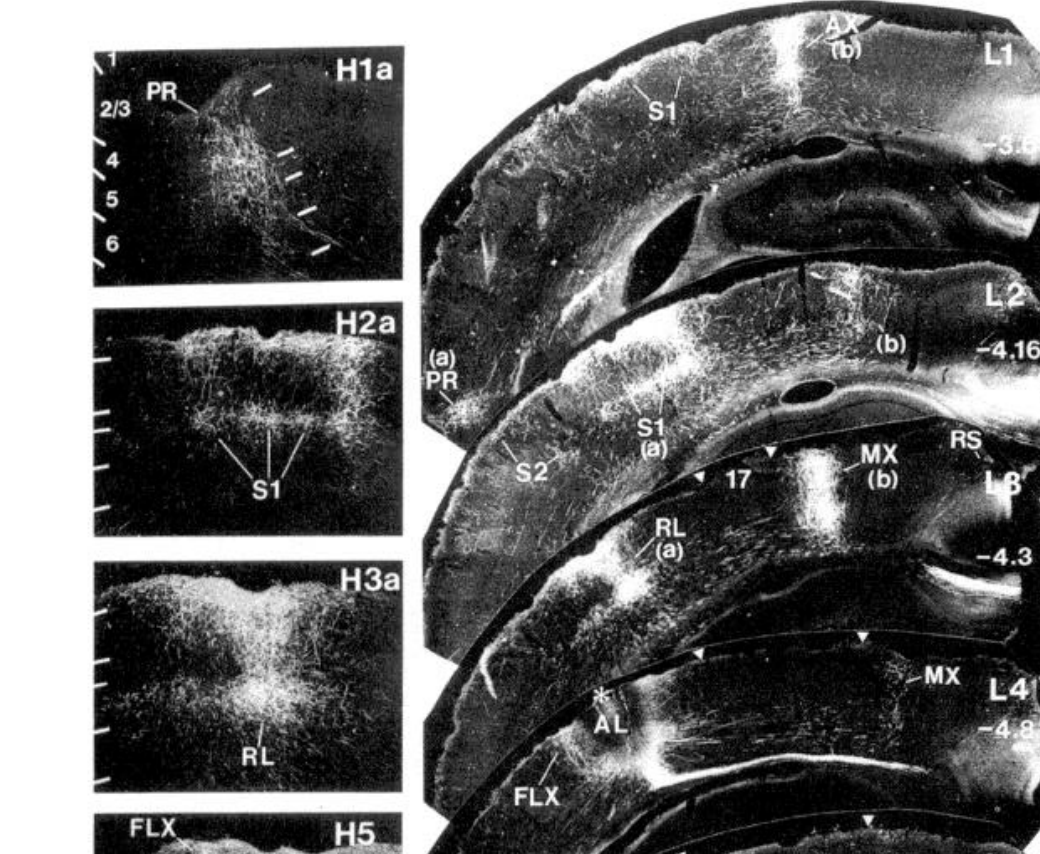

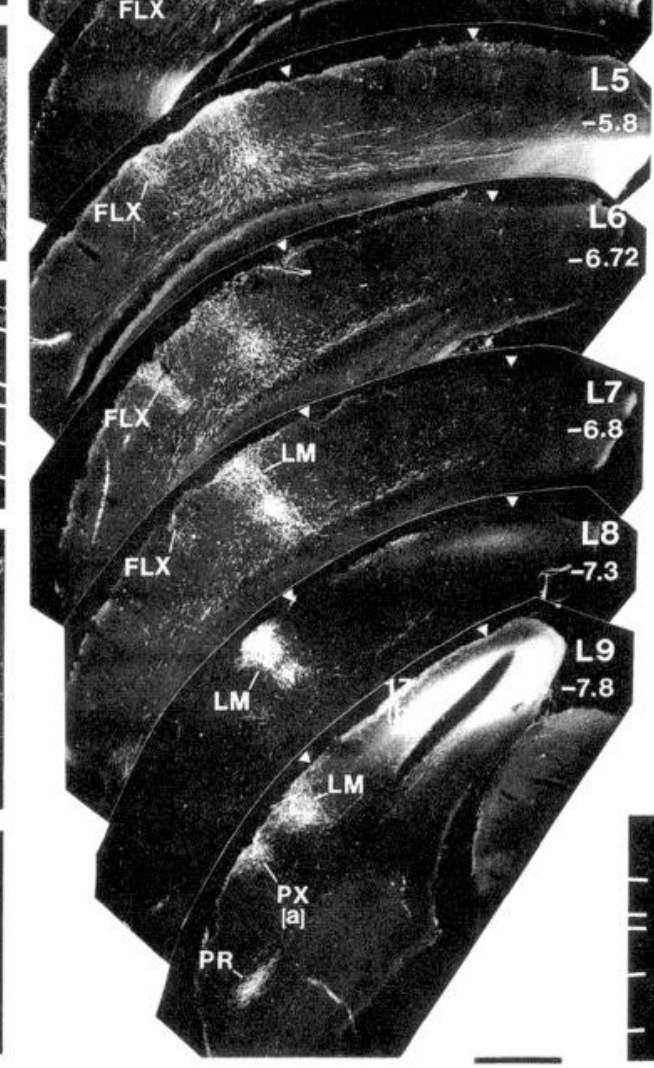

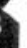
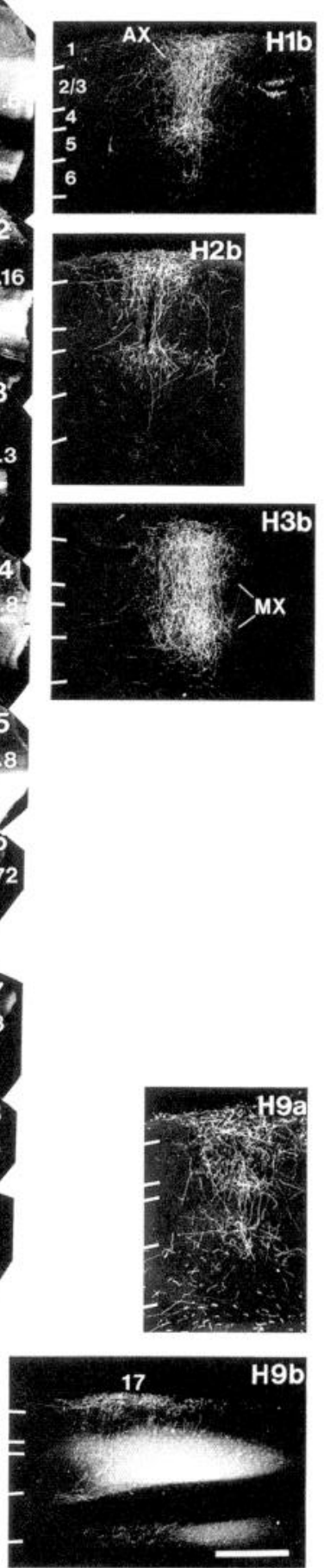

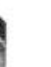

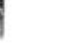
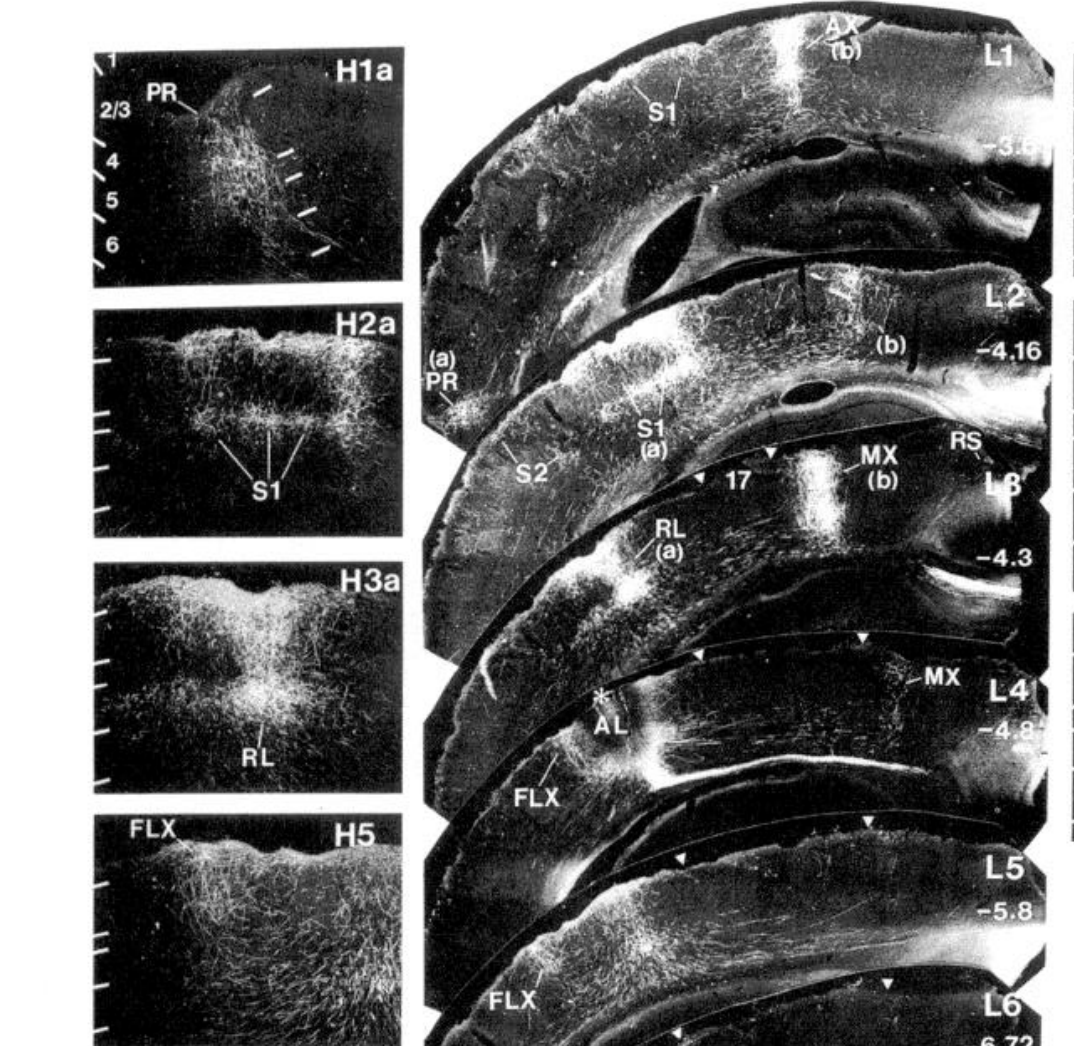

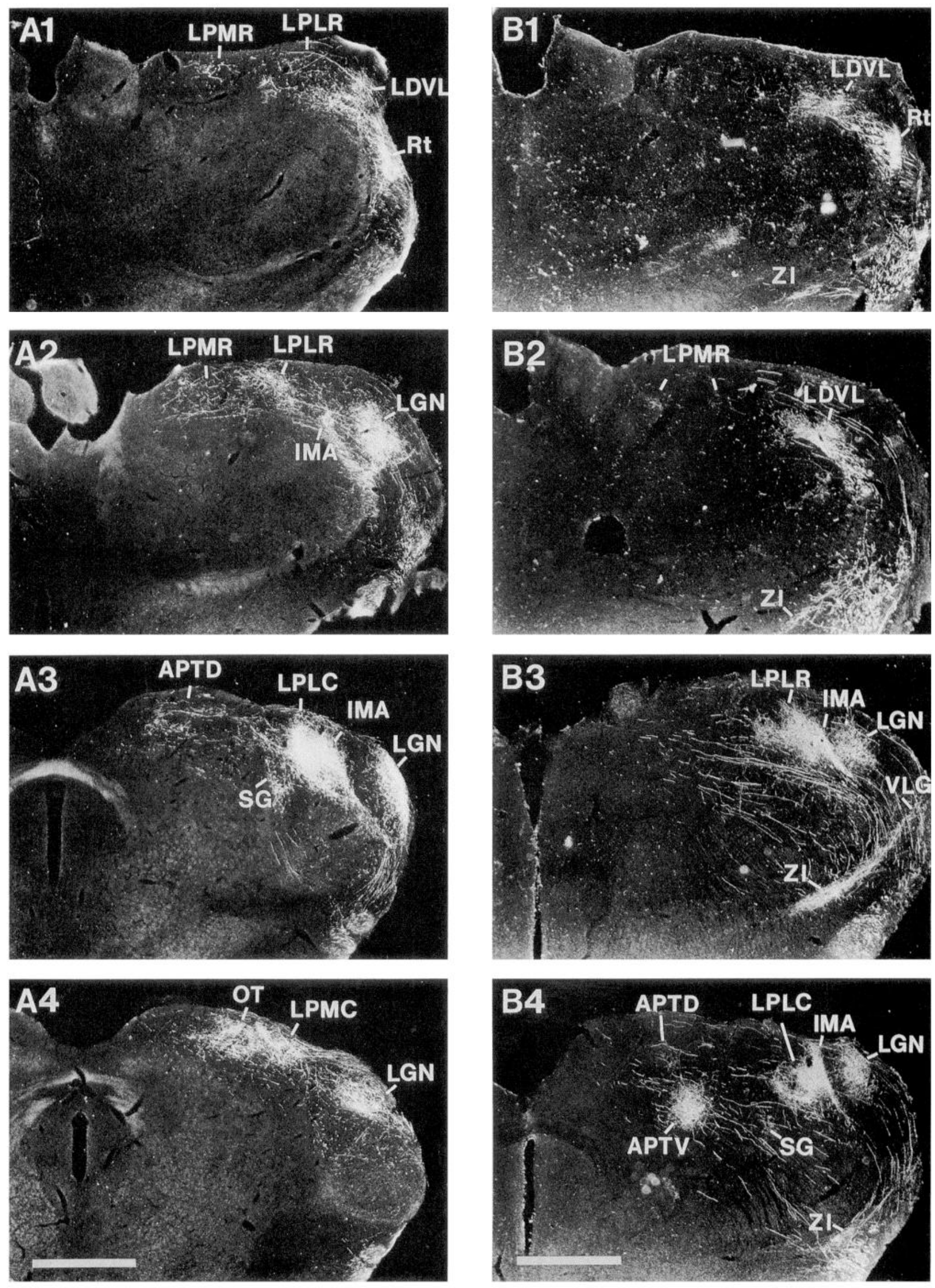
perficial and lower layers (Fig. 8H5); sites in posterior FLX, however, showed sparse projections to layer 4 (Fig. 8H6).

\section{Thalamic projections of $A L$ and $L M$}

Although our results on the topographic organization and the intracortical connectivity strongly suggest that LM and AL are distinct cortical areas, we sought further consolidation of this notion by establishing whether their projections to the thalamus are different. The results illustrated in Figure $9, A$ and $B$, show that this is indeed the case. Connections were found from both areas to a total of 14 targets of the thalamus. Of these, nine targets were shared. These included the lateral geniculate nucleus ( $L G N$ ), different subdivisions of the lateral posterior nucleus (LPMR, LPLR, LPLC), the ventrolateral subdivision of the laterodorsal nuclcus (LDVL), the reticular thalamic nucleus (Rt), the intermedullary thalamic nucleus (IMA), the suprageniculate nucleus (SG), and the dorsal portion of the anterior pretectal nucleus (APTD). Inputs to the mediocaudal subdivision of the lateral posterior nucleus (LPMC) came from LM only. Targets that were labeled after AL injections and were not labeled after LM injections included the zona incerta ( $\mathrm{ZI})$, the ventral geniculate nucleus (VLG), and the ventral subdivision of the anterior pretectal nucleus (APTV).

\section{Projections of $F L X$}

In a single case, a PHAL injection was made $7 \mathrm{~mm}$ lateral to the $\lambda$-point. Although the transcallosal labeling was incomplete, PHAL-labeled projections to cortex medial to the injection site and immediately lateral to area 17 strongly indicate that this represents an FLX injection. Near the injection site the projection was columnar and cxtrcmely densc (Fig. 10H3, L3). At morc anterior positions the fiber density decreased and the distribution appeared stratified (Fig. 10L2). As the projection progressed rostrally it appeared denser again and resumed a columnar appearance (Fig. $10 H 1, L I$ ). This pattern indicates two projections: one close to the injection site representing LM and the other representing $A L$ in anterior extrastriate cortex. The third set of projections within the circumstriate belt was located behind the injection site, was well separated from LM, and presumably corresponds to targets within PX (Fig. 10L4).

FLX is a known target of striate cortical projections (Olavarria and Montero, 1981, 1984; Malach, 1989) and it is therefore not surprising to see a reciprocal connection from FLX to area 17 (Fig. 10H4,L2-L4). Compared to the rest of the projections, inputs to MX were extremely weak (Fig. 10H2,L2) and mainly confined to layer $2 / 3$.

The laminar pattern of the area 17 projection was stratified, terminated principally in layers $1,2 / 3$, and 5 , and did not involve layer 4 . The projections to AL (Fig. 10HI) and LM (Fig. $10 H 3$ ) appeared as solid columns with terminal branches in all layers including layer 4 . This is interesting because the inputs from LM (Fig. 5) to FLX terminated in a similar laminar pattern, indicating that the reciprocal connections between LM and FLX have an identical organization.

\section{Projections of posterior $M X$}

The topographical organization of cortex medial to area 17 suggests the presence of two distinct visuotopic maps that share a common border: a caudally positioned area PM (posteromedial) and a more rostral area AM (anteromedial; Espinoza and Thomas, 1983). In a single case in which PHAL was injected 1.5 $\mathrm{mm}$ lateral to the $\lambda$-point, the injection site was recovered at the medial border of area 17 (Fig. 11L4). Labeling in striate cortex was seen in a swath of terminations that extended from AM to PL (Fig. $11 H 7 b, H 8, L 6-L 8$ ), corresponding to a large segment of the upper nasal and upper temporal visual field representation (Montero, 1981; Espinoza and Thomas, 1983) that maps at the anterior and posterior tip of AM and PM within MX (Espinoza and Thomas, 1983). The representation of the center of gaze lies near the boundary of these two areas (Espinoza and Thomas, 1983), and we have confirmed this by showing an appropriate input from area 17 (Fig. 4L2). In the case shown in Figure 11, this center of gaze projection was about $1.5 \mathrm{~mm}$ anterior to the injection site, which suggests that the injection was centered in posterior MX, a location that may correspond to PM.

In addition to the projection to area 17 (Fig. $11 L 5-L 8$ ), there were multiple projections to lateral, medial, and anterior cortical areas. Inputs to $\mathrm{LM}, \mathrm{AL}$, and $\mathrm{PX}$ were remarkably sparse and convincing terminal branching was seen only in LM (Fig. 11L6). In contrast, many more fibers ramified in FLX (Fig. 11L4-L7) and appeared to condense to several projection clusters within a region that extended $\approx 2 \mathrm{~mm}$ in the anteroposterior direction. Anterolaterally to this site, we identified inputs to an area in the lateral parictal cortex, which presumably corrcsponds to $\mathrm{S} 2$ (Fig. 11L2,L3). Input to medial and rostral cortex was extensive. The most prominent projections terminated in two sites within MX: one of these lay immediately in front of the injection site (Fig. 11H3,L3) and may correspond to AM; the other was seen $\approx 1.5 \mathrm{~mm}$ farther anteriorly (Fig. $11 H 2, L 2$ ). Substantial projections also terminated in frontal (Fig. $11 L I$ ) and cingulate cortex (Fig. 1 $1 \mathrm{Ll}$ ) and at multiple sites within agranular and granular RS (Fig. 11L3-L8). The projection to AX was sparse (Fig. $11 L 3$ ), and so were the projections to PR (Fig. 1 $1 L 7, L 8$ ) and the PS (Fig. 11L8). A dense input was also seen to the claustrum (not shown).

The laminar form of the projection to area 17 was stratified and dense terminations were seen in layers 1 and 6 (Fig. $11 H 6 c, H 8$ ). This pattern resembled that seen in striate cortex after injection of area 19 in the cat (Henry et al., 1991). It is important to note that layers $2 / 3$ and 5 were much less involved than in the striate cortical projections originating from LM (Fig. $5 H 3 b, H 4, H 5 b$ ) and $\mathrm{AL}$ (Fig. $8 H 9$ ), which also lacked terminations in layer 4 . The projections to LM (Fig. $11 \mathrm{H6a}$ ) terminated outside of layer 4 in layers 1,5 , and 6 . The termination pattern in FLX included mainly layers $1,2 / 3$, and 6, but sparser terminal branching often extended into layers 4 and 5 (Fig. $11 \mathrm{H} 4 a, H 6 b, H 7$ ). Projections to anterior sites of MX (Fig.

Figure 9. Corticothalamic projections of $\mathrm{LM}$ and $\mathrm{AL} . A 1-A 4$, Dark-field photomicrographs of serial coronal sections $(A 1$, most rostral; $A 4$, most caudal; medial, left; dorsal, toward top) showing PHAL-labeled projections to thalamus after PHAL injection into LM. Injection site was identified in posteromedial part of acallosal oval, adjoining the lateral border of area $17 . B 1-B 4$, Corticothalamic projections after PHAL injection into AL. Injection site was identified in anterior part of acallosal oval. Same format as $A$. For abbreviations, see Appendix. Scale bars, 1 mm. 

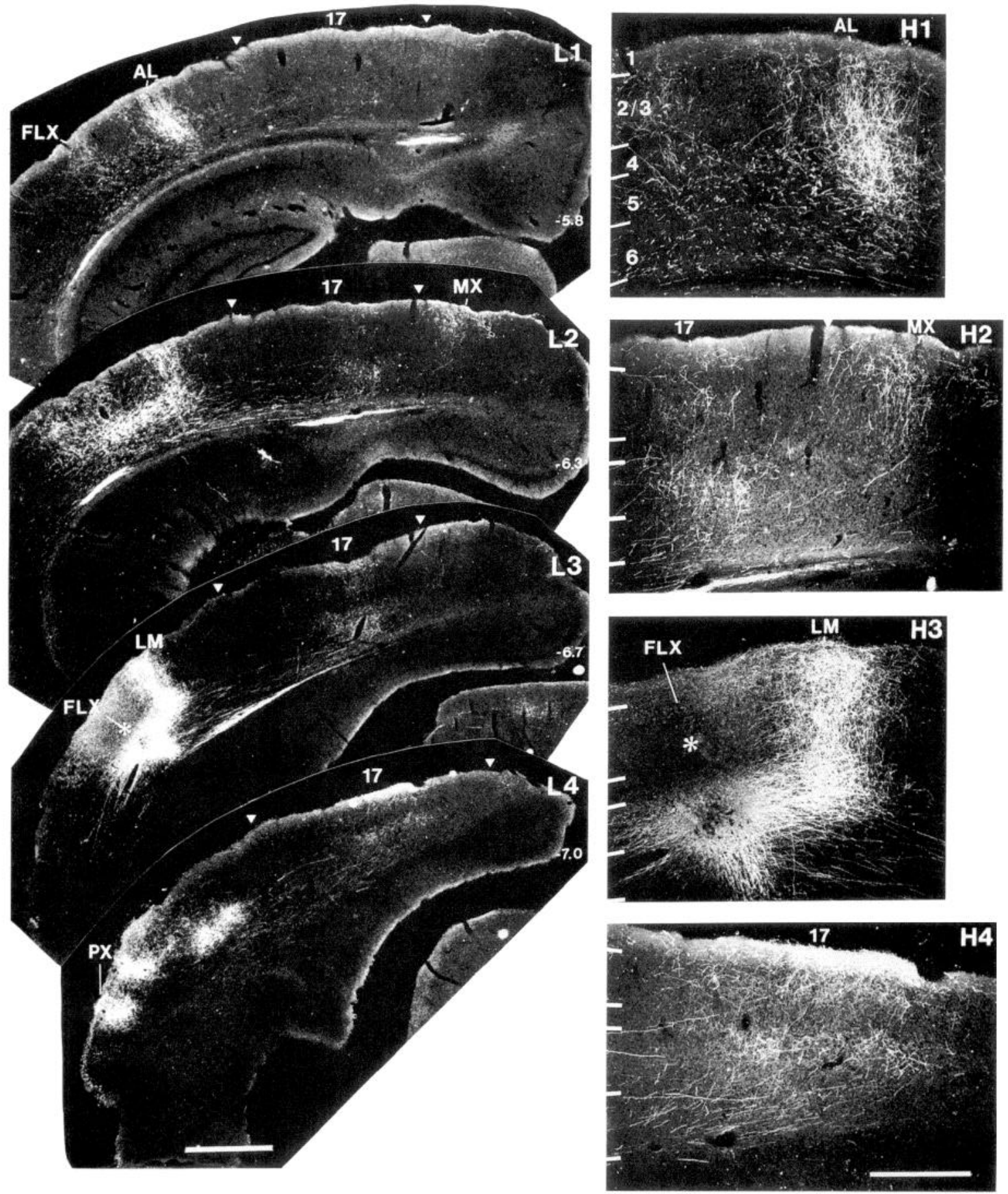

Figure 10. Laminar patterns of PHAL-labeled intracortical projections originating from the extrastriate FLX: reconstruction from serial coronal sections viewed under dark-field illumination. Injection site if indicated by asterisk. Format, orientation, and scales are the same as in Figure 2. For abbreviations, see Appendix.

$11 H 2, H 3$ ), frontal (Fig. 11H1), and cingulate cortex (Fig. 11HI) had a laminar form that did not precisely match the pattern of extrastriate cortical projections originating from area 17 . What they had in common with these projections were the terminations in layer 4 , but unlike the solid columns of striate cortical efferents, these projections from posterior MX had a much more stratified appearance and terminal branching was concentrated mainly in layer 1 , at the bottom of layer $2 / 3$, in layers 4 and 6 .

\section{Projections of anterior $M X$}

In a single case the PHAL injection was recovered rostromedially to area 17 within MX, at a site that presumably corresponds to area AM mapped by Espinoza and Thomas (1983). This assignment is based on the similarity in the location of the injection site with the MX projection labeled from central representations of area 17 (Fig. $4 L 2$ ). In addition, it is consistent 

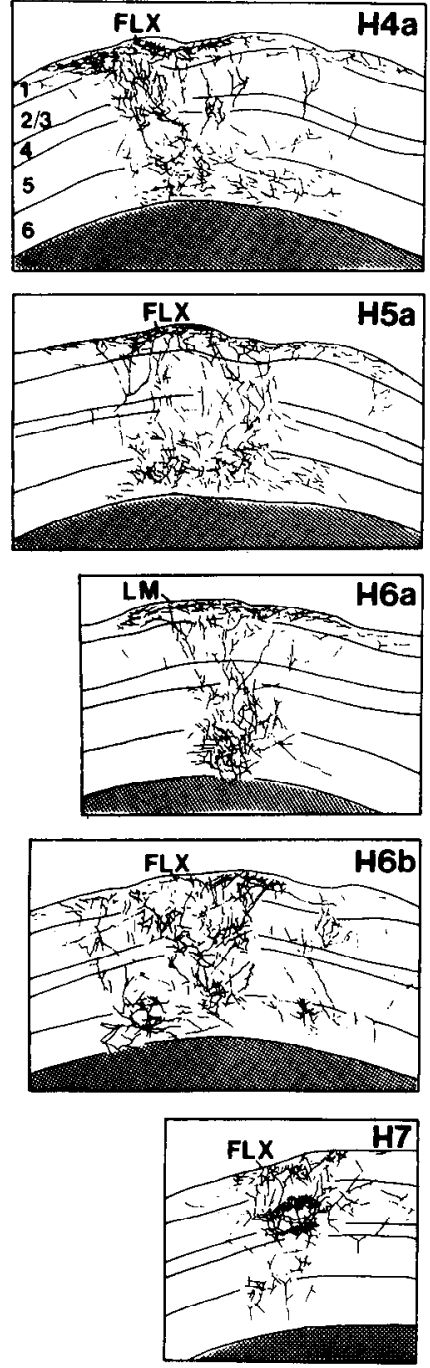

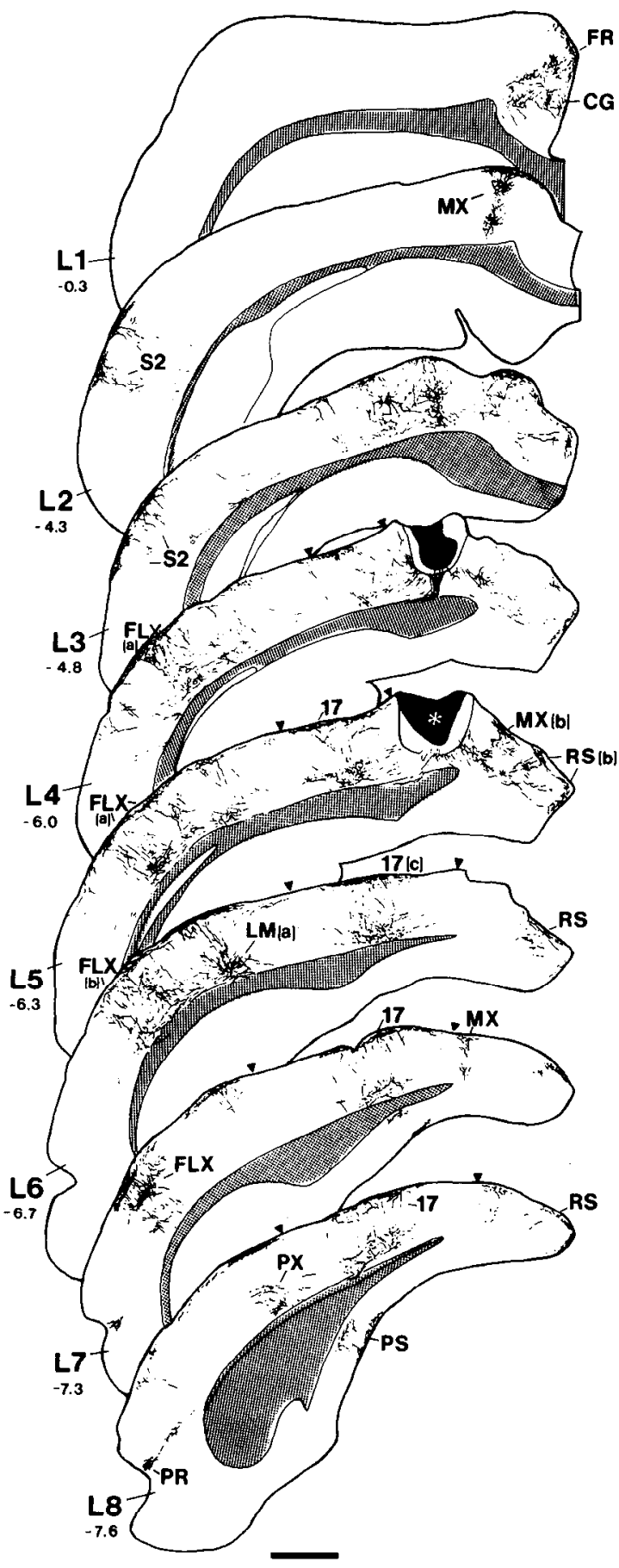

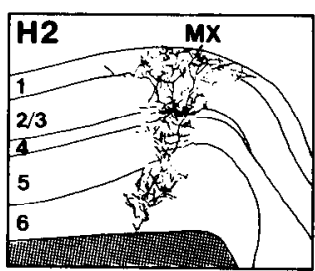
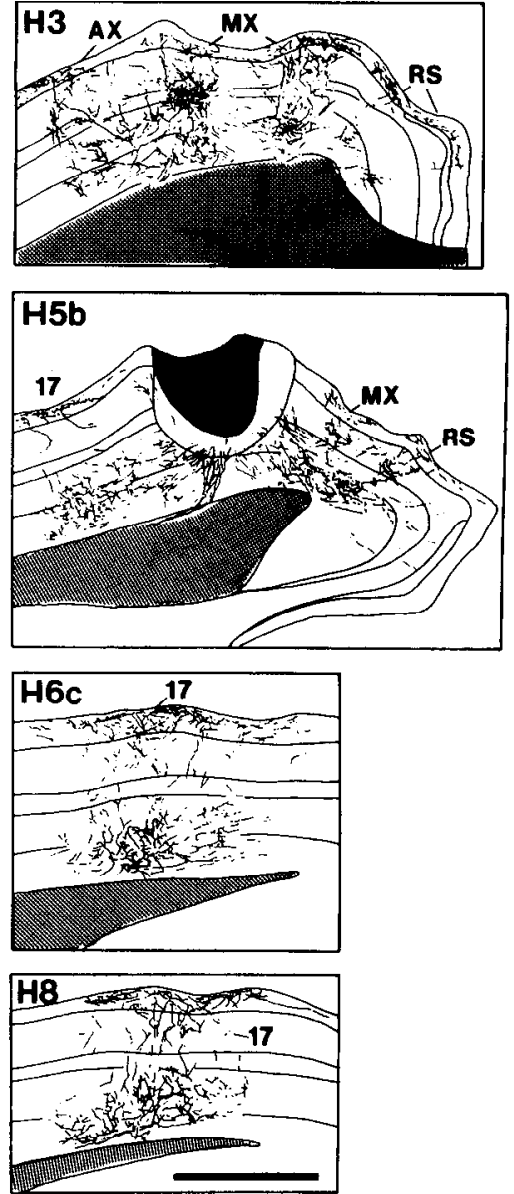

Figure 11. Laminar patterns of PHAL-labeled intracortical projections originating from posterior site within the extrastriate MX: camera lucida reconstruction from serial coronal sections. Injection site is indicated by asterisk. Format, orientation, and scales are the same as in Figure 2 . For abbreviations, see Appendix.

with the finding that the anterior MX injection labeled a single site in more anterior MX (Fig. 12L2), as opposed to the two sites labeled by the injection into posterior MX (Fig. 11L2,L3).

Outputs from anterior MX terminated in many of the same targets as outputs from posterior MX. The projections to area 17 were spread over a wide region that extended from the posterior edge to the anterior tip of striate cortex. Fibers in layer 1 showed the most extreme distribution, but inputs to the layer $3 / 4$ and layer $5 / 6$ borders were also widely distributed. In ad- dition, the projections were nonuniform along the rostrocaudal axis (Fig. 12L6-L8), which indicated that the input to striate cortex was clustered. Inputs to lateral cortex appeared more robust than after posterior $\mathrm{MX}$ injections and distinct projections were identified to LM (Fig. 12L9), AL (Fig. 12L5), and multiple sites within FLX (Fig. 12L4,L5,L8). Projections to areas in anterior and medial cortex terminated in AX (Fig. $12 L 2$ ), in sites in rostral MX (Fig. 12L2) and posterior MX (Fig. $12 \mathrm{H} 6 \mathrm{a}$ ), in posterior parts of frontal cortex (Fig. 12L1), 

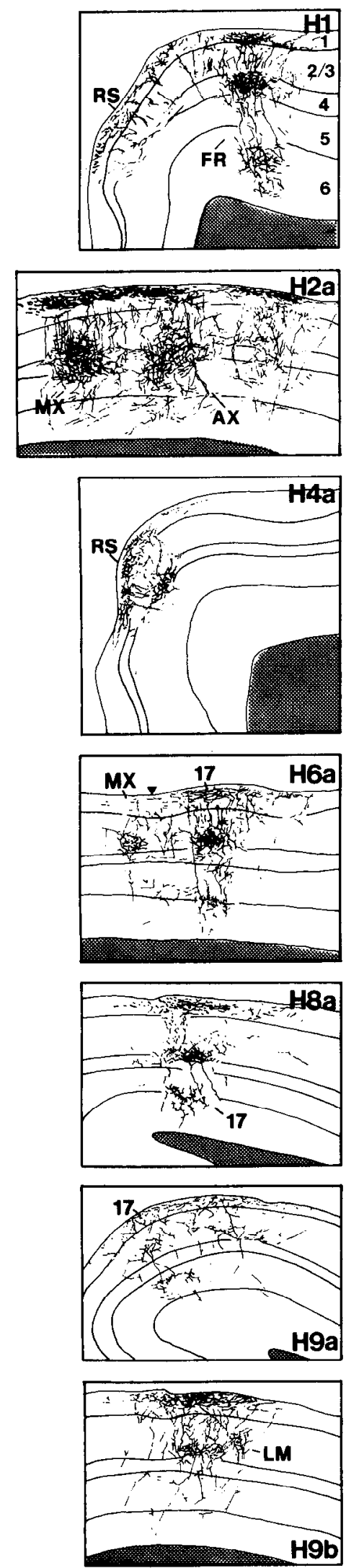
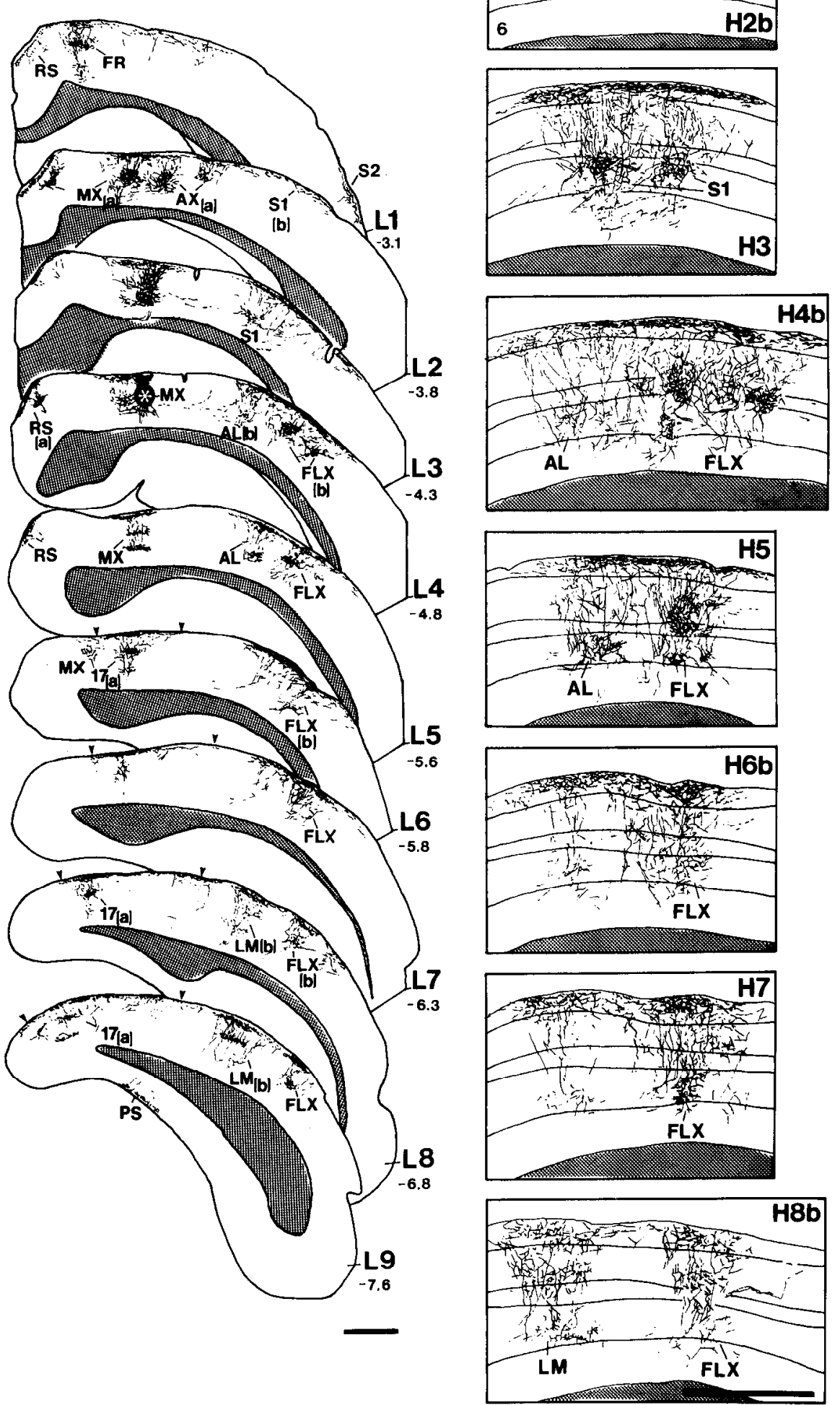

Figure 12. Laminar patterns of PHAL-labeled intracortical projections originating from anterior site within the extrastriate MX: camera lucida reconstruction from serial coronal sections. Injection site is indicated by asterisk. Format and scales are the same as in Figure 2 . Anterior is toward the top, medial to the left. For abbreviations, see Appendix. 
and in multiple targets within the granular and agranular RS (Fig. 12L1-L5). In contrast to posterior MX, anterior MX was sending a strong input to the primary somatosensory cortex (Fig. $12 L 3)$; the projection to the second somatosensory area, however, was relatively weak (Fig. 12L1). Because sections at the posterior and anterior pole of the brain were not immunostained, we cannot comment whether anterior MX provides inputs to PX and the cingulate cortex.

The results of anterior and posterior MX injections have shown that both regions project to many of the same cortical areas. Most interestingly, however, the lamination patterns of these projections differ. Unlike the bistratified appearance of the input to area 17 from posterior MX (Fig. $11 \mathrm{H6c}, \mathrm{H8}$ ), the striate cortical projections from anterior MX terminated in three strata: layer 1, and the borders of layers $3 / 4$ and 5/6 (Fig. 12H6a,H8a). An important feature of these projections was that inputs to the layer $3 / 4$ border were strong and that a substantial number of fibers ramified within layer 4 . A similar lamination pattern to that in area 17 was seen in LM (Fig. $12 \mathrm{H} 8 \mathrm{~b}$ ) and perhaps also in AL (Fig. 12H5). Again, the laminar pattern was clearly different from inputs provided by posterior MX (Fig. 11 H6a). Inputs to FLX, rostral MX (Fig. 12H2a), AX (Fig. 12H2a), and the frontal cortex (Fig. 12HI) were mainly directed to layer 4, but terminations typically also included layers $1,2 / 3,5$, or 6 , depending on the area under consideration. Similar to the projections from AL to the first and second somatosensory cortex, inputs from anterior MX terminated mainly in layers 1 and 5 (Fig. 12H3).

\section{Visual cortical projections to the superior colliculus}

The superior colliculus contains an orderly topographic representation of the contralateral visual hemifield (Siminoff et al., 1966). It receives input from the visual cortex and serves as a convenient map for assessing the topographic location of visual cortical injection sites. In the process of using this assay, we have noticed that different visual areas projected to different layers of the superior colliculus. After injection of the striate cortex, terminal ramifications were found focused in the superficial gray $(\mathrm{SuG})$ extending down to the border between the superficial and the optic layer (Op) (Fig. 13A). The focus of labeling after injections into LM was deeper in the tectum, involving mainly the Op and extending much sparser projections into deep parts of the SuG matter (Fig. 13B). Unlike the projection from area 17, input from LM was clustered. Injections of AL produced a distinctly different pattern from that of LM: terminals were confined to deep parts of the Op and inferior SuG (Fig. 13C). In all cases the terminal pattern was nonuniform, but in some cases clusters were more distinct than in others. A fourth type of projection pattern was seen after injections into posterior MX near the medial border of striate cortex. Here, labeled axons were distributed more widely and terminals ramified throughout $\mathrm{SuG}$ matter, the $\mathrm{Op}$, and inferior gray matter (Fig. 13D).

Figure 13. PHAL-labeled projections to superior colliculus: coronal sections viewed under dark-field illumination. $A$, Projection from area 17. $B$, Projection from area LM. $C$, Projection from area AL. $D$, Projection from posterior MX. Dorsal, top; medial, right. For abbreviations, see Appendix. Scale bars, $0.5 \mathrm{~mm}$.
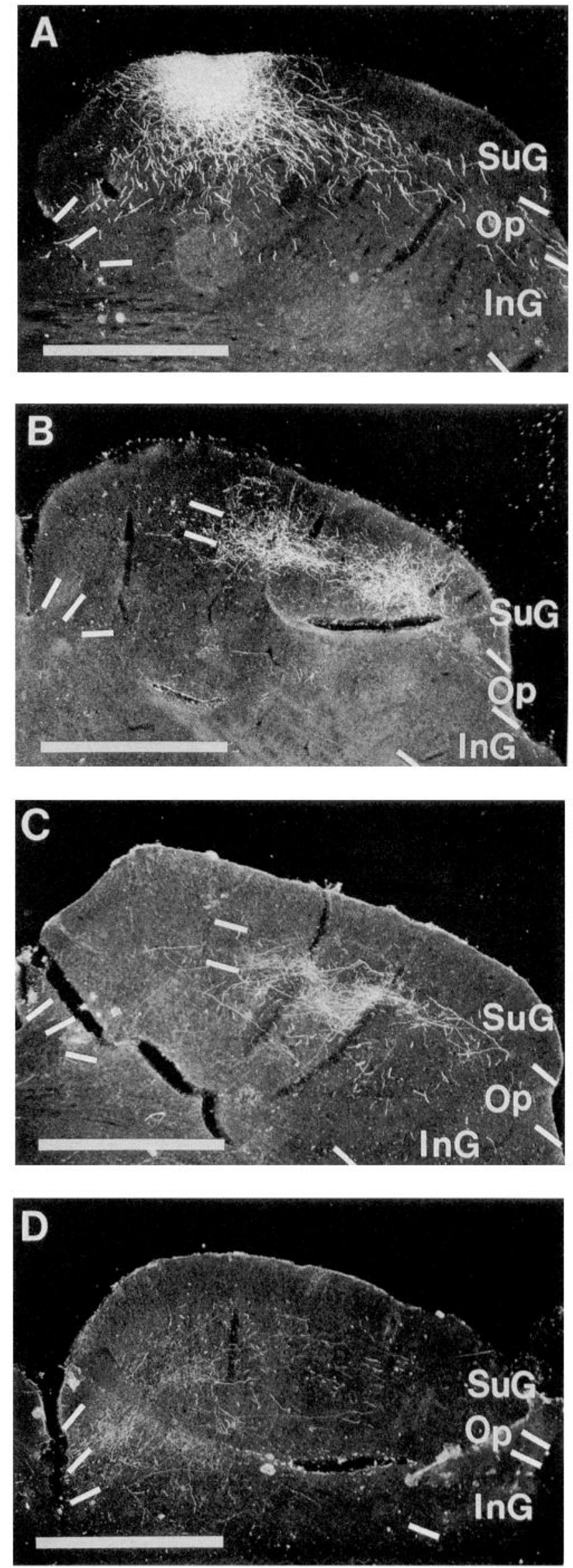


\section{Discussion}

Although several anatomical and physiological mapping studies have examined the rodent visual cortex, there is no consensus about its areal organization. One of the unresolved questions is how many visual areas border primary visual cortex. Arguments have been presented for several different schemes. At one end of the spectrum is Malach's (1989) view of a single area, analogous to the second visual area (V2) in primates. Other interpretations have postulated a smaller V2, restricted to the lateral border of striate cortex (Dräger, 1975; Tiao and Blakemore, 1976; Wagor et al., 1980; Kaas et al., 1989). A third set of observations suggests that extrastriate cortex adjoining the lateral border of area 17 contains as many as four distinct visual areas, and that two additional areas lie along the medial border (Espinoza and Thomas, 1983; Olavarria and Montero, 1984, 1989, 1990; Thomas and Espinoza, 1987; Spatz et al., 1991; Montero, 1993). The results provided in the present study are consistent with the third view and support the notion that striate cortex is bordered by several distinct areas. We have evidence for at least two areas adjoining the lateral border: LM and AL. Incomplete evidence suggests the presence of at least one more area within the PX region that adjoins the posterior border of striate cortex. Similar uncertainty exists about the number of areas within AX and MX in anterior and medial circumstriate cortex, but it seems reasonable to propose that $\mathrm{AX}$ contains at least a single area and that MX may have two distinct subdivisions. Our conclusions on the number of areas derive from the findings that (1) there are multiple regions that each represent large parts of the visual hemifield, (2) these regions project to different sets of cortical and subcortical targets, (3) projections to similar targets (cortex, superior colliculus) show different laminar patterns of terminations, and (4) different regions have asymmetrical reciprocal connections with each other. Similar criteria were used to define distinct areas in primate visual cortex (Van Essen, 1985). In our study, injections of 15 pairs of reciprocally connected areas showed asymmetrical laminar patterns in 14 pairs, and symmetrical patterns in the remaining pair. Based on these results, we conclude that areas in the rat visual cortex are organized in a multilevel hierarchy, similar to that proposed for the visual cortex of macaque monkey and cat (Felleman and Van Essen, 1991).

\section{Extrastriate areas}

Identification in coronal sections. Originally, areas $\mathrm{LM}$ and $\mathrm{AL}$ in rat cortex were identified as topographically organized territories contained within the anterior acallosal oval in lateral extrastriate cortex (Olavarria and Montero, 1984; Thomas and Espinoza, 1987). We have used callosal markers in the majority of our experiments, but in some cases transcallosal labeling was incomplete, forcing us to make areal assignments based on geographical position. While this may pose problems in gyrencephalic brains, the layout of areas in lissencephalic brains is extremcly stcrcotypcd (Olavarria ct al., 1987). Thus, in most cases it was straightforward to identify LM as the second to last striate recipient area of the lateral circumstriate belt, rostral to the posterior margin of the occipital pole (Fig. 3). Area AL was identified as the striate recipient zone immediately anterior to LM (Fig. 3). We have also used this indirect procedure in a number of cases for assigning injection sites to LM (Fig. 5) or AL. Without exception, the projection patterns observed in these cases were identical to those in which direct identification of the injected area was achieved through the presence of callosal markers (Fig. 8). Thus, we are confident that we have reliably identified areas LM, AL, and RL. For the identification of projection sites within the PX, FLX, AX, and MX it was more difficult to rely on relative positions, though it was easy to distinguish between the different regions. For this reason, we have described the projections to these subdivisions with provisional geographical terms such as PX, FLX, AX, and MX, until more complete information is available linking the visuotopic with the connectional organization. However, connectional evidence strongly suggests that the portion of MX lying at the medial border of area 17 is subdivided into an anterior and a posterior field that may correspond to areas AM and PM, respectively (Espinoza and Thomas, 1983): the anterior area, but not the posterior field, makes connections to the primary somatosensory cortex, and the two areas differ in the laminar organization of inputs to area 17, LM, and the superior colliculus (A. Burkhalter, unpublished observations).

Topography. Previous investigations of the organization of visual cortex in rat and squirrel (Kaas et al., 1989; Malach, 1989) have concluded that the multiple projections from striate cortex represent patches within a singlc arca, and that circumstriate cortex in rodents corresponds to area V2 of primates. This view is predicated on a reported trend that single points in striate cortex project to restricted parts of nearby extrastriate cortex (Malach, 1989). Our investigation does not substantiate this report. On the contrary, our results, as well as those of Olavarria and Montero (1984), show that (1) points in posterolateral striate cortex project to lateral, anterior, and medial parts of extrastriate cortex (Fig. 3), and (2) points in anteromedial area 17 project to medial, anterior, lateral, and posterior extrastriate cortex (Fig. 3).

Evidence for distinct areas is most complete for $\mathrm{LM}$ and $\mathrm{AL}$ in cortex lateral to area 17 . Physiological mapping of receptive fields in the acallosal oval of the lateral extrastriate cortex has shown that both striate recipient regions, AL and LM (Olavarria and Montero, 1984), correspond to distinct representations of the visual hemifield (Thomas and Espinoza, 1987; Spatz et al., 1991). Our results concur with this notion and show that AL and LM contain orderly maps of large parts of the visual field that include upper and lower quadrants including the center of gaze (Fig. 3). In addition, projections between LM and AL are organized as predicted from the topography of striate cortical projections: injections into anterior LM labeled projections to posterior AL (Fig. 5), and injections into anterior AL labeled projections in posterior LM (Fig. 8).

Projections. Strong support for distinct areas within lateral and medial extrastriate cortex also derives from our findings that different striate recipient sites are connected to different cortical targets. Area AL in lateral extrastriate cortex projects to primary and secondary somatosensory cortex (Fig. 8), while LM makes only sparse projections to S2 (Fig. 5; Sanderson et al., 1991). LM and AL each make strong projections to PR (Figs. 5, 8; Dcacon ct al., 1983), whereas PR is only a minor target of MX (Fig. 12L8). MX, on the other hand, is more intimately connected to frontal and cingulate cortex (Figs. 11, 12; Beckstead, 1979; Vogt and Miller, 1983; Miller and Vogt, 1984; Torrealba et al., 1984; Sesack et al., 1989) than LM and AL. Marked differences in the intracortical connectivity also exist between posterior and anterior subdivisions of MX: the projections from posterior MX to LM and AL are much weaker than those from anterior $\mathrm{MX}$, and only anterior MX makes a 


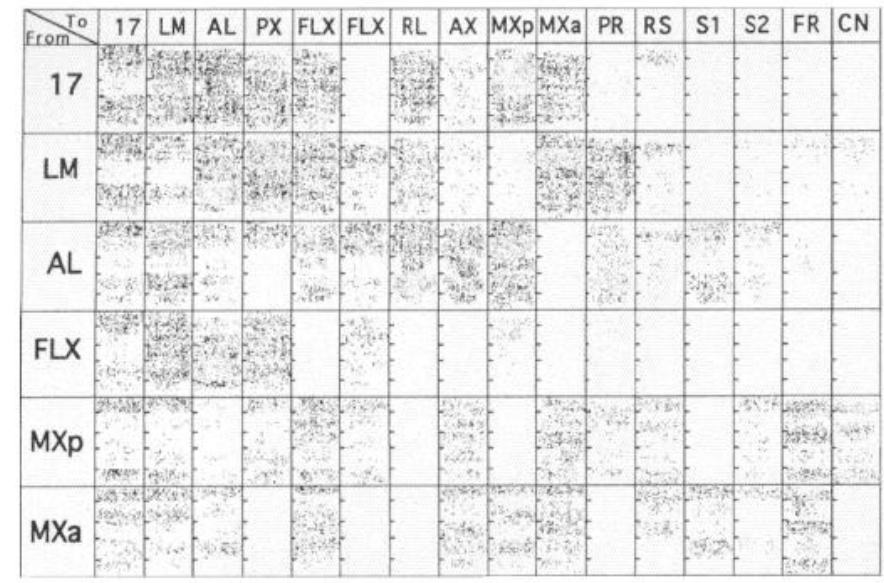

Figure 14. Summary diagram of laminar organization of projections between different visual areas in the rat cortex. The stippling represents terminal branching in different layers, which are indicated by tick marks on the left of each box. Layer 1 is at the top; layer 6 is at the bottom. The areas listed in the column on the left were injected with PHAL. The projection areas are listed in the top row. Notice that projections to FLX are represented in two boxes. This is to indicate the heterogeneity of laminar patterns seen in FLX. For abbreviations, see Appendix.

projection to primary somatosensory cortex (Figs. 11, 12). In addition, the projection to striate cortex from posterior $\mathrm{MX}$ is uniform, whereas that from anterior MX is clustered (Figs. 11, 12).

Further evidence that some of these areas are integrated into different circuits is provided by subcortical projections. Although corticothalamic projections from LM and AL share a number of common targets (e.g., LGN, LPMR, LPLR, LPLC, LDVL, Rt, IMA, SG, APTD), some of their outputs are distinct: LM makes strong projections to the nucleus of the optic tract and to LPMC, whereas AL projects to ZI, VLG, and APTV (Fig. 9). A recent study found that some of the common thalamic targets project back to extrastriate cortex (Sanderson et al., 1991). In addition it showed that the projection from the lateral posterior nucleus to $\mathrm{LM}$ is much stronger than that from AL. In agreement with our findings, these results were interpreted as evidence that $\mathrm{LM}$ and $\mathrm{AL}$ are distinct extrastriate areas.

There are also differences in the projections to the superior colliculus, where outputs from AL terminate below the inputs from LM (Fig. 13; Harvey and Worthington, 1990). In distinction to LM and AL, PX does not project to the superior colliculus (Harvey and Worthington, 1990; Burkhalter, unpublished observations). Corticotectal efferents from MX in medial extrastriate cortex occupy the deepest layers of the superior colliculus and terminate below the projections from LM and AL (Fig. 13), adding to the evidence of the distinctness of lateral and medial extrastriate cortex.

Support for independent areas within medial extrastriate cortex also derives from the laminar patterns of projections to areas 17 and LM. Both projections are of the feedback type and spare layer 4 (Coogan and Burkhalter, 1990). Projections from anterior and posterior MX share this basic pattern. However, the projections differ from each other in that anterior MX terminates in layers $1,2 / 3$, and 5 (Fig. $12 H 6 a, H 8 b$ ), whereas the input from posterior MX is restricted to layers 1 and 6 (Fig. $11 \mathrm{H} 6 \mathrm{a}, \mathrm{H} 6 \mathrm{c}$ ). Although it is possible that these differences are the result of unequal injections, labeling in the superior colliculus and the lateral posterior nucleus indicates that both injec-

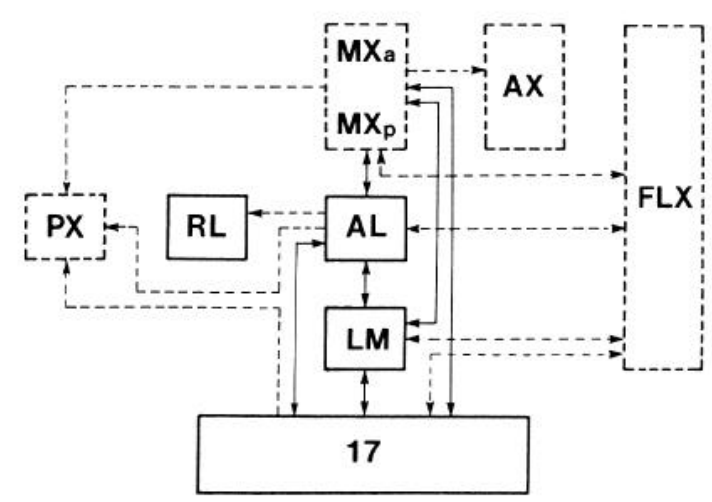

Figure 15. Hierarchy of visual areas. Solid boxes indicate identified visual areas. Broken boxes indicate cortical regions that are likely to be composed of multiple areas. Because of this, broken boxes may extend over several hierarchical levels. Solid lines indicate identified asymmetrical connections. Broken lines indicate symmetrical connections or that information is incomplete for classifying connections. For abbreviations, see Appendix.

tions extended at least to layer 5 (Sefton et al., 1981). Thus, it seems more likely that the different laminar projection patterns reflect projections from two different areas, which may correspond to the physiologically defined areas AM and PM (Espinoza and Thomas, 1983).

\section{Areal hierarchy}

Laminar patterns of reciprocal connections. The idea that hierarchical relationships in rat cortex can be discerned from the laminar organization of connections derives from the extensive analyses performed in primate visual cortex (Felleman and Van Essen, 1991). There, it was noticed that many reciprocal connections consist of forward projections terminating in layer 4 and feedback projections terminating outside of layer 4 . Such asymmetrical linkages are present between most reciprocally connected pairs of cortical areas.

In the rat, reciprocal intracortical connections share many of the features found in primate cortex. This analogy led to the proposal that laminar patterns can be used for distinguishing forward and feedback projections in rat visual cortex (Coogan and Burkhalter, 1990). However, unlike in primates, projections from striate cortex in rats and tree shrews (Sesma et al., 1984) are not restricted to layer 4 , and instead the projection is formed by a column of terminal branches that provide inputs to all cortical layers (Figs. 4H1,H2,H4,H6a,H8; $\mathrm{HI}, \mathrm{H} 3 \mathrm{~b}, \mathrm{H} 5 \mathrm{a}, \mathrm{H7}$; $8 H 1 b, H 3 b ; 11 H 2, H 3, H 7 ; 12 H 1, H 2 a, H 4 b, H 5 ; 14)$. Because of this feature, projections from striate cortex in rats more closely resemble the so-called lateral connections of primates, which are composed of two reciprocal legs with a similar laminar organization (Maunsell and Van Essen, 1983). Evidently, in such symmetrically connected pairs it is ambiguous whether one projection is forward and one is feedback, and because of this it is most reasonable to rank both areas at the same hierarchical level. In fact, we have evidence that columnar projections are involved in symmetrical relationships between areas LM and FLX (Figs. 5, 11). As a consequence, columnar projections in rat can be identified as forward-going only if the reciprocal leg shows a different lamination pattern that excludes layer 4 , that is to say, if the connections within a pair are asymmetrical. With this rule it is possible to rank areas relative to each other: areas at the lowest level of the hierarchy make only forward projections and all the projections they receive are feedback. The only 
area that embodies all of these attributes is the principal cortical target of the LGN (Ribak and Peters, 1975), area 17. Striate cortex, therefore, occupies the first hierarchical level (Fig. 15). This is in agreement with the findings in primates (Maunsell and Van Essen, 1983) and is supported by reports from cats and tree shrews that extrastriate cortical inputs to striate cortex typically exclude layer 4 (Lund et al., 1979; Rockland et al., 1982; Symonds and Rosenquist, 1984; Henry ct al., 1991).

Using a combination of callosal and geographical markers, we have identified at least three pairs of reciprocally connected areas: area 17 and $\mathrm{LM}$, area 17 and $\mathrm{AL}$, and $\mathrm{LM}$ and $\mathrm{AL}$. The identification of 12 additional pairs-area 17/posterior $\mathrm{MX}$, area 17/anterior $\mathrm{MX}$, area $17 / \mathrm{FLX}, \mathrm{LM} /$ posterior $\mathrm{MX}, \mathrm{AL}$ / posterior MX, LM/anterior MX, AL/anterior MX, FLX/LM, FLX/AL, FLX/anterior MX, FLX/posterior MX, and anterior $\mathrm{MX} /$ posterior $\mathrm{MX}$ - is more tentative, because the multiple projection sites within FLX and MX have not yet been definitively assigned to specific areas, and because it therefore remains questionable whether true reciprocal pairs have been studied. In addition, we have analyzed only single cases of injection into anterior MX and posterior MX, but our analysis of injections into these areas in developing brains supports the results reported here (T. A. Coogan and A. Burkhalter, unpublished observations).

Without exception, projections from striate cortex to LM and AL include terminals in layer 4 (Fig. 14). By contrast, projections that return from LM and AL to area 17 spare layer 4 (Fig. 14), and thus the reciprocal connections form asymmetrical pairs. Because LM makes feedback projections to area 17 and all of its remaining projections are forward, it occupies the second hierarchical level (Fig. 15). AL ranks at a higher level, because it makes feedback projections to LM and area 17 and the reciprocal projections from these areas are forward (Figs. 14, 15).

Although we were easily able to identify the RL area, its place in the hierarchy is ambiguous because we have information about its inputs only. All of these are columnar and include layer 4 (Fig. 14). Since in our scheme an area cannot rank lower than the hierarchically highest area that provides columnar input, we have provisionally placed RL at the level of AL.

At present, the inclusion of some of the less firmly established areas into the hierarchy is more tentative, because their reciprocal relationships to other areas have not been studied exhaustively. Nevertheless, it is tempting to exploit the known connectivity patterns for this purpose. With these qualifications in mind, the asymmetrical projections between MX and LM and $\mathrm{AL}$ suggest that the anterior and posterior sites within $\mathrm{MX}$ rank above LM and AL (Figs. 14, 15). For the same reason the anterior subdivision that may correspond to the AM area of Espinoza and Thomas (1983) ranks hierarchically above posterior MX (Fig. 15), which may correspond to the physiologically identified area PM (Espinoza and Thomas, 1983).

It has been reported that higher visual cortical areas of cats and primates project to deeper layers of the superior colliculus than hierarchically lower areas (cf. Felleman and Van Essen, 1991; Harting et al., 1992; see also Cusick, 1988). Although there are exceptions to this rule, our results support this notion and show that areas that rank at progressively higher levels of the hierarchy (17-LM-AL-MX) project to progressively deeper layers of the superior colliculus (Figs. 13, 15; Harvey and Worthington, 1990). Additional support for ranking MX above LM and $\mathrm{AL}$ derives from its relatively stronger connections with areas of higher-order processing in frontal and cingulate cortex
(Fig. 14; Beckstead, 1979; Vogt and Miller, 1983; Miller and Vogt, 1984; Sesack et al., 1989).

By far the most difficult problem is how to integrate FLX into the hierarchical scheme. FLX receives columnar connections from areas at three different hierarchical levels and makes columnar projections to $\mathrm{LM}$ and $\mathrm{AL}$, leading to conflicting areal assignments. We conclude, therefore, that FLX is composed of arcas that are at different hicrarchical levels. Given the feedback projection from AL, one site in FLX must be below AL, whereas other sites, those that receive columnar projections from MX, must be above AL, as high as the level of anterior MX. Area AL being above an FLX site is an exception to the organization in cats and primates where hierarchically higher areas are farther away from the striate cortex (Felleman and Van Essen, 1991).

Ranking of PX is difficult because we have information about its inputs only and no information about the laminar organization of its outputs (Fig. 14). All of the inputs, except for the feedback projection from MX (Fig. $11 L 8$ ), are of the columnar form and in isolation provide no unambiguous information about hierarchical relationships. The feedback input from MX, however, suggests that PX ranks below MX (Fig. 15). It is conceivable that PX occupies a separate level between AL and MX, but it is equally likely that PX ranks at the same level as $\mathrm{AL}$ or that some subdivisions occupy levels below that.

Similar to PX, information about the connectivity of $A X$ is restricted to inputs, which all include layer 4 (Fig. 14). This is true even for inputs from MX (Fig. 12H2a), which suggests that AX ranks at least at the same level with $\mathrm{MX}$, but is conceivably hierarchically higher. Its lower limits could extend as low as to the level occupied by $\mathrm{AL}$, because the columnar input (Fig. $8 H 1 b$ ) from AL may be lateral and not forward.

Areas in RS, frontal cortex, PR, and cingulate cortex are likely higher in the hierarchy. However, our current knowledge of the lamination of these connections does not provide the means to include them in the hierarchy. Similarly, the somatosensory areas are not included in the hierarchy. Although they would appear to rank below AL and LM, because there are no direct connections with the striate cortex, their position relative to primary visual cortex cannot be defined.

Consideration of projection types. The possibility that columnar projections are involved in either symmetrical or asymmetrical reciprocal relationships raises the question of whether projections with comparable laminar organization play similar functional roles. In this context it would be of interest to know whether all columnar projections contact the same postsynaptic targets, that is, whether their inputs are directed to similar projection systems. If the connections involved in symmetric and asymmetric relationships are equivalent, one could argue that the hierarchical organization of cortical areas in rat is based on half steps. In this species, feedback projections could be reciprocated by a projection type also involved in lateral relationships, whereas in the macaque monkey feedback projections are strictly paired with forward projections.

The question of whether projections with the same laminar form have identical function can also be raised in relation to the similarities between feedback and long-range horizontal projections within striate (Fig. 4H6b; Rockland and Pandya, 1979; Burkhalter, 1989; Burkhalter and Charles, 1990) and extrastriate cortex (e.g., LM, Fig. 5L5,L6). This may suggest that feedback projections and intrinsic long-range projections contact similar targets. In addition, it could indicate that feedback projections, similar to long-range intrinsic connections, link disparate points 
of the visuotopic map (Gilbert and Wicsel, 1983). At first glance, this fits the observation that input from LM to striate cortex is more widespread than the projection from area 17 to LM (Figs. 4,5 ). However, LM is small compared to area 17 and the proportion of the visuotopic map covered by an injection into LM is larger than that of an area 17 injection, making it difficult to compare the topographic precision of forward and feedback projections. Also, feedback projections to area 17 are more widespread in layers 1 and 5 than in layer $2 / 3$ (Fig. $5 H 4, H 5$ ), suggesting laminar differences in the mapping of inputs. Alternatively, it is possible that the different extent of the projection fields is unrelated to topographic mapping and instead reflects the subcellular disposition of inputs to distal versus proximal dendrites on the target cell.

\section{Different stages of processing}

In primates, different hierarchical levels correspond to different stages of visual processing (Felleman and Van Essen, 1991), and recent evidence suggests that this functional organization is reflected in the cortical output to the superior colliculus. Cusick (1988) has shown that in squirrel monkey the corticotectal projections of areas V2, MT, and DL (V4 of macaque), which represent higher processing stages than $\mathrm{V} 1$, terminate in deeper layers than striate cortex. We have seen a similar organization of the corticotectal output in rat visual cortex, where striate cortical projections terminate in superficial layers (Fig. 13A) and projections of extrastriate areas from increasingly higher hierarchical levels terminate in progressively deeper layers (Fig. $13 B-D$ ). More direct evidence for a hierarchy of processing stages derives from measurements of receptive field size. They are smallest in striate cortex, larger in LM and AL, and largest in the FLX (Espinoza and Thomas, 1983). Although little is known about the response properties of neurons in different extrastriate areas in rodents, cells in AX respond to visual and vibrissal stimuli (Wagor et al., 1980). Processing of polysensory information is commensurate with an elevated position of $\mathrm{AX}$ in the cortical hierarchy.

\section{Evolution of visual areas}

The presence of multiple visual areas adjoining rat striate cortex raises the question of how these areas relate to a single area V2 that surrounds most of the striate cortex of many other animals. Several phylogenetic sequences can be imagined: (1) a large ancestral area was broken up into separate areas in rat cortex, (2) creation of $\mathrm{V} 2$ de novo or by the merger of smaller areas, and (3) enlargement of a small area. The first scenario seems unlikely, because increasingly visual animals, comparing squirrels (Kaas et al., 1989), cats (Rosenquist, 1985), and primates (Felleman and Van Essen, 1991), have a single V2 that occupies increasingly more of the outline of striate cortex. (Though because of location and modularity of intrinsic organization these regions have been called $\mathrm{V} 2$ in different species, it is unclear whether these areas can indeed be considered homologous.) At present there is no convincing evidence to indicate whether the second or the third possibility is more likely. What seems clear, however, is that even within the phylogeny of rodents (Wood, 1985), evolution gravitated toward a cortical organization that allows processing of different visual functions within a single cortical area, rather than in several distinct areas. The reason for this may lie in the pressure to preserve close neighborhood relationships so that computations can be performed locally and neuronal wiring is minimized (Durbin and Mitchison, 1990).

\section{Appendix}

Abbreviations

$17 \quad$ Area 17

18a Cytoarchitectonic subdivision $18 \mathrm{a}$

18b Cytoarchitectonic subdivision $18 \mathrm{~b}$

Al Primary auditory cortex

AL Anterolateral area

AM Anteromedial area

APTD Anterior pretectal nucleus, dorsal

APTV Anterior pretectal nucleus, ventral

AX Anterior complex

$\mathrm{CG}, \mathrm{CN} \quad$ Cingulate cortex

d Lamina dissicans

DYS Dysgranular cortex

ENT Entorhinal cortex

FLX Far lateral complex

FR Frontal cortex

IMA Intermedullary thalamic area

InG Inferior gray

LDVL Laterodorsal thalamic nucleus, ventrolateral

LGN Lateral geniculate nucleus

LM Lateromedial area

LPLC Lateral posterior thalamic nucleus, laterocaudal

LPLR Lateral posterior thalamic nucleus, laterorostral

LPMC Lateral posterior thalamic nucleus, mediocaudal

LPMR Lateral posterior thalamic nucleus, mediorostral

MX Medial complex

MXa Anterior medial complex

MXp Posterior medial complex

Op Optic nerve layer superior colliculus

OT Nucleus of the optic tract

pe Lamina principalis externa

pi Lamina principalis interna

PM Posteromedial area

PR Perirhinal cortex

PS Presubiculum

PX Posterior complex

RL Rostrolateral area

RS Retrosplenial cortex

Rt Reticular thalamic nucleus

S1 Primary somatosensory cortex

S2 Secondary somatosensory cortex

SG Suprageniculate nucleus

SuG Superficial gray layer superior colliculus

VLG Ventral geniculate nucleus

ZI Zona incerta

\section{References}

Andersen RA, Asanuma C, Essick G, Siegel RM (1990) Corticocortical connections of anatomically and physiologically defined subdivisions within the inferior parietal lobule. J Comp Neurol 296:65-1 13.

Beckstead RM (1979) An autoradiographical examination of corticocortical and subcortical projections of the mediodorsal-projections (prefrontal) cortex in the rat. J Comp Neurol 184:43-62.

Boussad D, Ungerleider LG, Desimone R (1990) Pathways for motion analysis: cortical connections of the medial superior temporal and fundus of the superior temporal visual areas in the macaque. J Comp Neurol 296:462-495.

Burkhalter A (1989) Intrinsic connections of rat primary visual cortex: laminar organization of axonal projections. J Comp Neurol 279:171186.

Burkhalter A, Charles V (1990) Organization of local axon collateral of efferent projection neurons in rat visual cortex. J Comp Neurol 302:920-934.

Coogan TA, Burkhalter A (1990) Conserved patterns of cortico-cortical connections define areal hierarchy in rat visual cortex. Exp Brain Res 80:49-53.

Cusick CG (1988) Anatomical organization of the superior colliculus in monkeys: corticotectal pathways for visual and visuomotor functions. Prog Brain Res 75:1-15.

Cusick CG, Lund RD (1981) The distribution of the callosal projection to the occipital visual cortex in rats and mice. Brain Res 214:239259. 
Deacon TW, Eichenbaum H, Rosenberg P, Eckmann KW (1983) Afferent connections of the perirhinal cortex in the rat. J Comp Neurol 220:168-190.

DeYoe EA, Van Essen DC (1988) Concurrent processing stream in monkey visual cortex. Trends Neurosci 11:219-226.

Dräger UC (1975) Receptive fields of single cells and topography in mouse visual cortex. J Comp Neurol 160:269-290.

Durbin R, Mitchison G (1990) A dimension reduction framework for understanding cortical maps. Nature 343:644-647.

Espinoza SG, Thomas HC (1983) Retinotopic organization of striate and extrastriate visual cortex in the hooded rat. Brain Res 272:137144.

Felleman DJ, Van Essen DC (1991) Distributed hierarchical processing in the primate cerebral cortex. Cereb Cortex $1: 1-47$.

Fricdman DP, Murray EA, O'Ncill JB, Mishkin M (1986) Cortical connections of the somatosensory fields of the lateral sulcus of macaques: evidence for a corticolimbic pathway for touch. J Comp Neurol 252:323-347

Gerfen CR, Sawchenko PE (1984) An anterograde neuroanatomical tracing method that shows the detailed morphology of neurons, their axons, and terminals: immunocytochemical localization of an axonally transported lectin, Phaseolus vulgaris leucoagglutinin (PHAL). Brain Res 290:219-238

Gilbert CD, Wiesel TN (1983) Clustered intrinsic connections in cat visual cortex. J Neurosci 3:1116-1133.

Gilbert CD, Wiesel TN (1989) Columnar specificity of intrinsic horizontal and corticocortical connections in cat visual cortex. J Neurosci 9:2432-2442.

Harting JK, Updyke BV, Van Licshout DP (1992) Corticotcctal projections in the cat: anterograde transport studies of twenty-five cortical areas. J Comp Neurol 324:379-414.

Harvey AR, Worthington DR (1990) The projection from different visual cortical areas to the rat superior colliculus. J Comp Neurol 298:281-292

Henry GH, Salin PA, Bullier J (1991) Projections from areas 18 and 19 to cat striate cortex: divergence and laminar specificity. Eur J Neurosci 3:186-200.

Kaas JH (1980) A comparative survey of visual cortex organization in mammals. In: Comparative neurology of the telencephalon (Ebbeson S, ed), pp 483-502. New York: Plenum.

Kaas JH, Krubitzer LA, Johanson KL (1989) Cortical connections of areas 17 (V-I) and 18 (V-II) of squirrels. J Comp Neurol 281:426446.

Krieg WJS (1946) Connections of cerebral cortex. I. The albino rat. B. Structure of the cortical areas. J Comp Neurol 84:277-323.

Lund JS, Henry GH, MacQueen CL, Harvey AR (1979) Anatomical organization of the primary visual cortex (area 17) of the cat. A comparison with area 17 of the macaque monkey. J Comp Neurol 184:599-618.

Malach R (1989) Patterns of connections in the rat visual cortex. J Neurosci 9:3741-3752.

Maunsell JHR, Van Essen DC (1983) The connections of the middle temporal visual area (MT) and their relationship to a cortical hierarchy in the macaque monkey. J Neurosci 3:2563-2586.

Mesulam M-M (1978) Tetramethylbenzidine for horseradish peroxidase neurochemistry: a non-carcinogenic blue reaction-product with superior sensitivity for visualizing neural afferents and efferents. J Histochem Cytochem 26:106-117.

Miller MW, Vogt BA (1984) Direct connection of rat visual cortex with sensory, motor, and association cortices. J Comp Neurol 226: 184-202.

Montero VM (1981) Comparative studies on the visual cortex. In: Cortical sensory organization, Vol 2, Multiple visual areas (Woolsey $\mathrm{CN}$, ed), pp 33-81. Clifton, NJ: Humana.

Montero VM (1993) Retinotopy of cortical connections between the striate cortex and extrastriate visual areas in the rat. Exp Brain Res, in press.

Montcro VM, Rojas A, Torrcalba F (1973a) Retinotopic organization of striate and peristriate visual cortex in the albino rat. Brain Res 53: 197-201.

Montero VM, Bravo H, Fernandez V (1973b) Striate-peristriate cortico-cortical connections in the albino and gray rat. Brain Res 53: 202-207.

Nauta WJH, Bucher VM (1954) Efferent connections of the striate cortex of the albino rat. J Comp Neurol 100:257-281.
Olavarria J, Montero VM (1981) Reciprocal connections between the striate cortex and extrastriate cortical visual areas in the rat. Brain Res 217:358-363.

Olavarria J, Montero VM (1984) Relation of callosal and striateextrastriate cortical connections in the rat: morphological definition of extrastriate visual areas. Exp Brain Res 54:240-252.

Olavarria J, Montero V (1989) Organization of visual cortex in the mouse revealed by correlating callosal and striate-extrastriate connections. Vis Neurosci 3:59-69.

Olavarria J, Montero V (1990) Elaborate organization of visual cortex in the hamster. Neurosci Res 8:40-47.

Olavarria J, Malach R, Van Sluyters RC. (1987) Development of visual callosal connections in neonatally enucleated rats. J Comp Neurol 260:321-348.

Paxinos G, Watson C (1986) The rat brain in stereotaxic coordinates, 2d ed. Sydney: Academic.

Ribak CE, Peters A (1975) An autoradiographic study of the projections from the lateral geniculate body of the rat. Brain Res 92:341368.

Rockland KS, Pandya DN (1979) Laminar origins and terminations of cortical connections of the occipital lobe in the thesus monkey. Brain Res 179:3-20.

Rockland KS, Lund JS, Humphrey AL (1982) Anatomical banding of intrinsic connection in striate cortex of tree shrews (Tupaia glis). J Comp Neurol 209:41-58.

Rosenquist AC (1985) Connections of visual cortical areas in the cat. In: Cerebral cortex, Vol 3 (Peters A, Jones EG, eds), pp 81-111. New York: Plenum.

Sanderson KJ, Dreher B, Gaycr N (1991) Prosenccphalic connections of striate and extrastriate areas of rat visual cortex. Exp Brain Res 85:324-334.

Sefton AJ, Mackay-Sim A, Baur LA, Cottee LJ (1981) Cortical projections to visual centers in the rat: an HRP study. Brain Res 215:113.

Sesack SR, Deutch AY, Roth RH, Bunney BS (1989) Topographical organization of the efferent projections of the medial prefrontal cortex in the rat: an anterograde tract-tracing study with Phaseolus vulgaris leucoagglutinin. J Comp Neurol 290:213-242.

Sesma MA, Casagrande VA, Kaas JH (1984) Cortical connections of area 17 in tree shrews. J Comp Neurol 230:337-351.

Siminoff R, Schwassman HO, Kruger L (1966) An electrophysiological study of the visual projection to the superior colliculus of the rat. J Comp Neurol 127:435-444.

Spatz WB, Vogt DM, Illing R-B (1991) Delineation of the striate cortex and the striate-peristriate projections in the guinea pig. Exp Brain Res $84: 495-504$

Symonds LL, Rosenquist AC (1984) Cortico-cortical connections among visual areas in the cat. J Comp Neurol 229:1-38.

Thomas HC, Espinoza SG (1987) Relationships between interhemispheric cortical connections and visual areas in hooded rats. Brain Res 417:214-224

Tiao Y.C, Blakemore C (1976) Functional organization in the visual cortex of the golden hamster. J Comp Neurol 168:459-482.

Torrealba F, Olavarria J, Carrasco MA (1984) Cortical connections of the anteromedial extrastriate visual cortex in the rat. Exp Brain Res 56:543-549.

Van Essen DC (1985) Functional organization of primate visual cortex. In: Cerebral cortex, Vol 3 (Peters A, Jones EG, eds), pp 259-329. New York: Plenum.

Van Essen DC, Newsome WT, Maunsell JHR, Bixby JL (1986) The projections from striate cortex to areas $\mathrm{V} 2$ and $\mathrm{V} 3$ in the macaque monkey: asymmetries, areal boundaries, and patchy connections. J Comp Neurol 244:451-480.

Vogt BA, Miller MW (1983) Cortical connections between rat cingulate cortex and visual, motor, and postsubicular cortices. J Comp Neurol 216:192-210

Wagor E, Mangini NJ, Pearlman AL (1980) Retinotopic organization of striate and extrastriate visual cortex in the mousc. J Comp Ncurol 193:187-202.

Wood AE (1985) In: Evolutionary relationships among rodents: a multidisciplinary analysis (Luckett WP, Hartenberger J-L, eds), pp 475-509. New York: Plenum. 